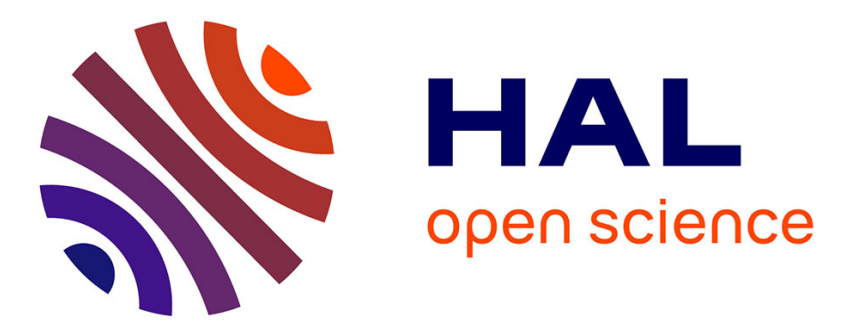

\title{
Quadratic solid-shell elements for nonlinear structural analysis and sheet metal forming simulation
}

Peng Wang, Hocine Chalal, Farid Abed-Meraim

\section{To cite this version:}

Peng Wang, Hocine Chalal, Farid Abed-Meraim. Quadratic solid-shell elements for nonlinear structural analysis and sheet metal forming simulation. Computational Mechanics, 2017, 59 (1), pp.161-186. 10.1007/s00466-016-1341-8 . hal-02385962

\section{HAL Id: hal-02385962 https://hal.science/hal-02385962}

Submitted on 29 Nov 2019

HAL is a multi-disciplinary open access archive for the deposit and dissemination of scientific research documents, whether they are published or not. The documents may come from teaching and research institutions in France or abroad, or from public or private research centers.
L'archive ouverte pluridisciplinaire HAL, est destinée au dépôt et à la diffusion de documents scientifiques de niveau recherche, publiés ou non, émanant des établissements d'enseignement et de recherche français ou étrangers, des laboratoires publics ou privés. 


\title{
Quadratic solid-shell elements for nonlinear structural analysis and sheet metal forming simulation
}

\author{
Peng WANG, Hocine CHALAL, Farid ABED-MERAIM \\ LEM3, UMR CNRS 7239 - Arts et Métiers ParisTech, 4, rue Augustin Fresnel, 57078 Metz \\ Cedex 03, France
}

\begin{abstract}
In this paper, two quadratic solid-shell (SHB) elements are proposed for the threedimensional modeling of thin structures. These consist of a twenty-node hexahedral solid-shell element, denoted SHB20, and its fifteen-node prismatic counterpart, denoted SHB15. The formulation of these elements is extended in this work to include geometric and material nonlinearities, for application to problems involving large displacements and rotations as well as plasticity. For this purpose, the SHB elements are coupled with large-strain anisotropic elastoplastic constitutive equations for metallic materials. Although based on a purely threedimensional approach, several modifications are introduced in the formulation of these elements to provide them with interesting shell features. In particular, a special direction is chosen to represent the thickness, along which a user-defined number of integration points are located. Furthermore, for efficiency requirements and for alleviating locking phenomena, an in-plane reduced-integration scheme is adopted. The resulting formulations are implemented into the finite element software ABAQUS/Standard and, to assess their performance, a variety of nonlinear benchmark problems are investigated. Attention is then focused on the simulation of various complex sheet metal forming processes, involving large strain, anisotropic plasticity, and doublesided contact. From all simulation results, it appears that the SHB elements represent an interesting alternative to traditional shell and solid elements, due to their versatility and capability of accurately modeling selective nonlinear benchmark problems as well as complex sheet metal forming processes.
\end{abstract}

Keywords finite element, quadratic solid-shell, thin structures, nonlinear analysis, anisotropic plasticity, sheet metal forming. 


\section{Introduction}

Nowadays, the numerical modeling has become an indispensable simulation tool in many fields of the industry, such as automotive, aerospace, and civil engineering. The finite element (FE) method, a widespread numerical tool, provides great assistance to engineers in the design of products and optimization of manufacturing processes. Despite the growing development of computational resources, reliability and efficiency of the FE analysis remain important features in the simulation practice. The present work deals with the simulation of thin structures, which is conventionally achieved using classical shell and continuum solid elements. However, in some circumstances, traditional shell and solid elements suffer from various locking phenomena, such as membrane locking, thickness locking, shear locking, etc. In addition, shell elements are often not appropriate for the modeling of complex sheet metal forming processes involving doublesided contact, partly due to the use of plane-stress assumptions in their formulation. To remedy these shortcomings, considerable effort has been devoted to the development of solid-shell elements during the last few decades. The key idea behind this original concept of solid-shell elements is to combine the advantages of both FE technologies, namely shell and continuum formulations. The main benefits of this solid-shell concept may be summarized as follows: easier formulation, based on a purely three-dimensional approach, with displacements as the only degrees of freedom; consideration of fully three-dimensional constitutive laws, with no planestress restrictions; direct calculation of thickness variations; natural treatment of double-sided contact, thanks to the availability of actual top and bottom surfaces; 3D modeling of thin structures, using only a single element layer and few integration points, while accurately describing the through-thickness phenomena.

Most solid-shell elements developed in the literature are based on the reduced-integration technique (see, e.g., Zienkiewicz et al. [1]). In the case of linear interpolation, this consists most often in adopting an in-plane one-point quadrature rule, while considering a number of integration points along the thickness. In addition to the reduced-integration scheme, several other numerical strategies, such as the assumed strain method (ASM), the enhanced assumed strain (EAS) approach, the assumed natural strain (ANS) concept, were developed in the literature to eliminate various kinds of locking phenomena (see, e.g., [2-17]). Note that, for linear under-integrated solid-shell elements, special stabilization procedures are required for the control 
of zero-energy (hourglass) modes, which are induced by the reduced-integration rule (see, e.g., Abed-Meraim and Combescure [12], Schwarze et al. [18]).

In this paper, two quadratic solid-shell elements are proposed for the 3D nonlinear analysis of thin structures. These formulations are extended to include geometric and material nonlinearities, following the earlier works on the family of SHB elements. The first solid-shell element in this family was developed by Abed-Meraim and Combescure [6], and consists of an eight-node hexahedral element denoted SHB8PS. Its formulation was subsequently improved by AbedMeraim and Combescure [12], especially in terms of locking reduction, while the hourglass modes were efficiently controlled by implementing a new stabilization procedure. The performance of the SHB8PS element was demonstrated through a representative set of selective benchmark tests as well as sheet metal forming processes involving large strains, anisotropic plasticity, and contact (see Abed-Meraim and Combescure [12], Salahouelhadj et al. [19]). Then, a six-node prismatic solid-shell element denoted SHB6 was developed by Trinh et al. [20], as a complement to the SHB8PS element for the modeling of complex geometries whose meshing requires the combination of hexahedral and prismatic elements. Although the performance of the SHB6 is good in the whole, its convergence rate remains slower than that of the SHB8PS, and requires finer meshes to obtain accurate solutions. More recently, the quadratic counterparts of the above hexahedral and prismatic solid-shell elements were developed by Abed-Meraim et al. [21], in order to improve the overall performance and convergence rate. These quadratic versions consist of a twenty-node hexahedral element, denoted SHB20, and a fifteen-node prismatic element, denoted SHB15. Likewise, their formulation is based on a fully three-dimensional approach with an in-plane reduced-integration rule. The performance of these elements has been evaluated by Abed-Meraim et al. [21] within the framework of small strain and elastic benchmark problems. In the present work, however, the formulation of the quadratic SHB15 and SHB20 elements is extended to the framework of large displacements and rotations. Moreover, the resulting formulations are coupled with large-strain anisotropic elasto-plastic constitutive equations, which allows modeling complex and challenging structural problems, such as sheet metal forming processes.

The remainder of the paper is outlined as follows. The general formulation of the quadratic solid-shell elements, SHB15 and SHB20, is presented in Section 2. Then, the performance of these elements is assessed in Section 3, first through a variety of linear and nonlinear benchmark 
problems. In Section 4, the proposed formulations are applied to the simulation of complex sheet metal forming processes, including springback, incremental forming, and deep drawing tests. Finally, the main conclusions and remarks are drawn in Section 5.

\section{Basic formulations for the quadratic solid-shell elements}

Despite some differences between the prismatic and hexahedral solid-shell elements (e.g., in terms of geometry, interpolation functions, etc.), their theoretical formulations show a number of similarities. In this section, a general formulation, common to both SHB15 and SHB20 solid-shell elements, is introduced. This formulation, which was previously developed by AbedMeraim et al. [21] within the framework of small strains, is extended here to the large-strain framework.

\subsection{Geometry and integration points}

Figure 1 illustrates the reference geometry and location of integration points for the SHB15 and SHB20 solid-shell elements. The starting point for the formulation of these quadratic solid-shell elements is the classical 3D approach, used for conventional quadratic continuum elements, with fifteen nodes for the prismatic SHB15 element and twenty nodes for the SHB20 element. Then, a special direction $\zeta$ (see Fig. 1) is chosen as the thickness direction, along which a user-defined number of integration points are arranged. In the $\xi-\eta$ plane corresponding to each $\zeta$-coordinate of these through-thickness integration points, are defined a total number of three integration points for the prismatic SHB15 element, and four integration points for the hexahedral SHB20 element, as shown in Fig. 1. The coordinates and associated weights of these integration points can be obtained using the classical Gauss distribution method (see, e.g., Zienkiewicz et al. [22]). It is worth noting that, in the case of full integration for conventional quadratic solid elements, three in-plane integration points with three through-thickness integration points are required for prismatic elements, while nine in-plane integration points with three through-thickness integration points are used for hexahedral elements. 


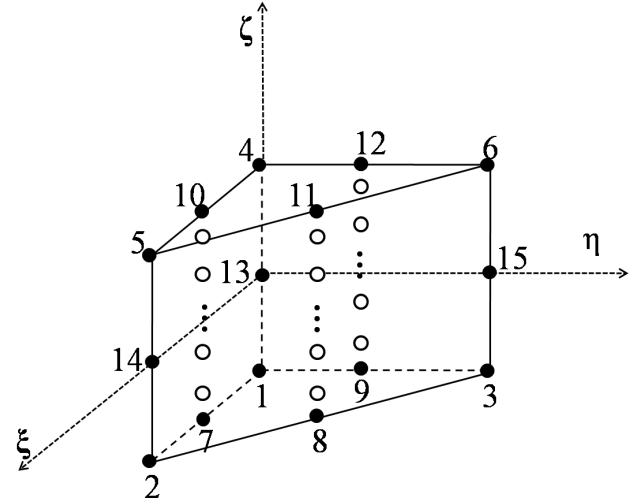

(a) SHB15

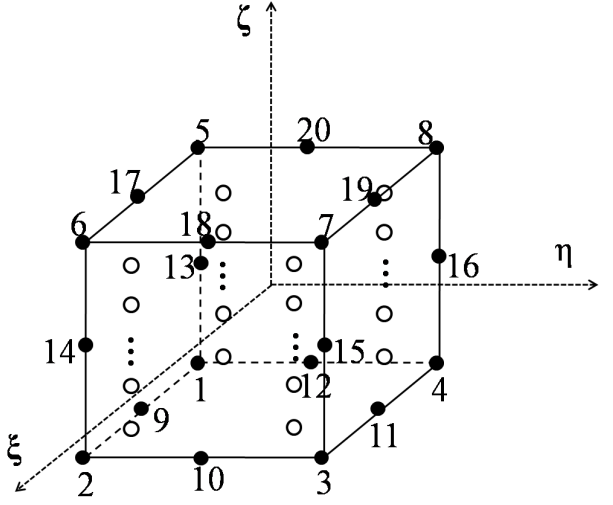

(b) SHB20

Fig. 1. Reference geometry and location of integration points for the SHB15 and SHB20 solid-shell elements.

Moreover, to provide the proposed elements with some desirable shell features and to reduce locking, special local element frames are introduced, which are attached to the element midplanes associated with each integration point. In these local physical coordinate systems, associated with the $\zeta$-coordinate of each integration point, the fully three-dimensional elasticity tensor of the material is specified. Note that this is a first major difference with conventional continuum elements, for which no such local element frames are considered. Figure 2 illustrates, in the case of the SHB20 element, the definition of these local element frames, which are built using the following procedure. First, the element mid-plane corresponding to a given integration point $k$ is defined using the physical nodal coordinates, which is represented in Fig. 2 by the four points $\mathrm{P}_{1}^{k}, \mathrm{P}_{2}^{k}, \mathrm{P}_{3}^{k}$ and $\mathrm{P}_{4}^{k}$. These latter points allow us, in turn, to define four mid-points $\mathrm{m}_{12}^{k}$, $\mathrm{m}_{23}^{k}, \mathrm{~m}_{34}^{k}$ and $\mathrm{m}_{41}^{k}$, which are the barycenters of $\left(\mathrm{P}_{1}^{k} \mathrm{P}_{2}^{k}\right),\left(\mathrm{P}_{2}^{k} \mathrm{P}_{3}^{k}\right),\left(\mathrm{P}_{3}^{k} \mathrm{P}_{4}^{k}\right)$ and $\left(\mathrm{P}_{4}^{k} \mathrm{P}_{1}^{k}\right)$, respectively. Then, the first base vector, $\mathbf{e}_{1}^{k}$, of the local coordinate system is defined as being parallel to $\left(\mathrm{m}_{41}^{k} \mathrm{~m}_{23}^{k}\right)$, while the second vector $\mathbf{e}_{2}^{k}$ is defined parallel to $\left(\mathrm{m}_{12}^{k} \mathrm{~m}_{34}^{k}\right)$. Vector $\mathbf{e}_{2}^{k}$ is modified by adding a correction term $\mathbf{e}_{c}^{k}$, so that vectors $\mathbf{e}_{1}^{k}$ and $\left(\mathbf{e}_{2}^{k}+\mathbf{e}_{c}^{k}\right)$ are orthogonal, which gives 
$\mathbf{e}_{c}^{k}=-\frac{\left(\mathbf{e}_{1}^{k}\right)^{T} \cdot \mathbf{e}_{2}^{k}}{\left(\mathbf{e}_{1}^{k}\right)^{T} \cdot \mathbf{e}_{1}^{k}} \mathbf{e}_{1}^{k}$.

Finally, the third base vector $\mathbf{e}_{3}^{k}$ is simply obtained by the following cross-product (see Fig. 2):

$\mathbf{e}_{3}^{k}=\mathbf{e}_{1}^{k} \times\left(\mathbf{e}_{2}^{k}+\mathbf{e}_{c}^{k}\right)$

The same strategy is applied to the prismatic SHB15 element, in order to define the associated local coordinate systems, and is not repeated here for conciseness.

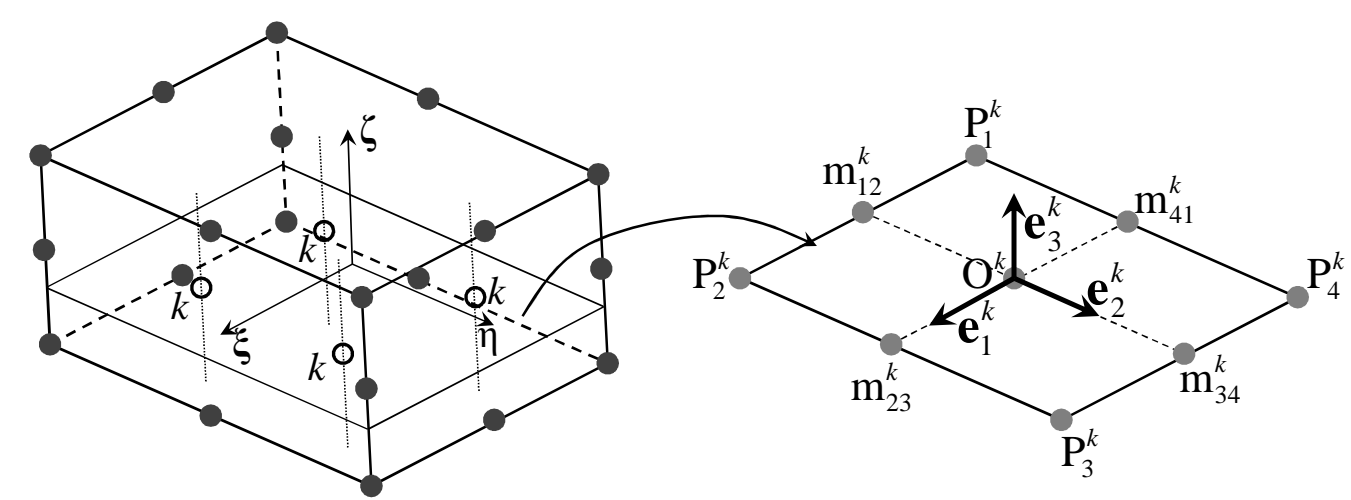

Fig. 2. Schematic representation of the local element frame associated with the $k$ th integration point of the SHB20 element.

\subsection{Quadratic interpolation for the SHB elements}

Using the classical isoparametric approach, the SHB15 and SHB20 solid-shell elements adopt the conventional shape functions $N_{I}$ for quadratic prismatic and hexahedral elements, respectively. The spatial coordinates $x_{i}$ and the displacement field $u_{i}$ within the element are expressed as functions of the nodal coordinates and the nodal displacement, respectively

$$
\begin{aligned}
& x_{i}=x_{i I} N_{I}(\xi, \eta, \zeta)=\sum_{I=1}^{n} N_{I}(\xi, \eta, \zeta) x_{i I}, \\
& u_{i}=d_{i I} N_{I}(\xi, \eta, \zeta)
\end{aligned}
$$


where the lowercase subscript $i$ varies from 1 to 3, and represents the spatial coordinate directions, while the uppercase subscript $I$ goes from 1 to $n$, with $n$ being the number of element nodes ( $n=15$ for the SHB15 element, and $n=20$ for the SHB20 element). Note that in Eq. (4) above, the convention of implied summation over repeated indices has been used, which will be also adopted in the sequel.

\subsection{Strain-displacement relationship and discrete gradient operator}

Based on the interpolation of the displacement field (Eq. (4)), the linear part $\varepsilon$ of the strain tensor is defined by the following relationship:

$\varepsilon_{i j}=\frac{1}{2}\left(u_{i, j}+u_{j, i}\right)=\frac{1}{2}\left(d_{i I} N_{I, j}+d_{j I} N_{I, i}\right)$.

The combination of Eqs. (3) and (4), along with the expression of the shape functions $N_{I}(\xi, \eta, \zeta)$, allows us to develop the displacement field in the following form:

$u_{i}=a_{0 i}+a_{1 i} x_{1}+a_{2 i} x_{2}+a_{3 i} x_{3}+c_{1 i} h_{1}+c_{2 i} h_{2}+\cdots+c_{\alpha i} h_{\alpha}$,

where $h_{\alpha}$ are functions of the nodal coordinates $\xi, \eta, \zeta$, in the reference coordinate system, and $\alpha$ varies from 1 to 11 for the SHB15 element, and from 1 to 16 for the SHB20 element. For the SHB15 element, the $h_{\alpha}$ functions are expressed as follows:

$\left\{\begin{array}{l}h_{1}=\xi \zeta, h_{2}=\eta \zeta, h_{3}=\xi \eta, h_{4}=\xi \eta \zeta, h_{5}=\xi^{2}, h_{6}=\eta^{2}, h_{7}=\zeta^{2}, \\ h_{8}=\xi^{2} \zeta, h_{9}=\eta^{2} \zeta, h_{10}=\xi \zeta^{2}, h_{11}=\eta \zeta^{2},\end{array}\right.$

while for the SHB20 element, they are given by

$\left\{\begin{array}{l}h_{1}=\xi \zeta, h_{2}=\eta \zeta, h_{3}=\xi \eta, h_{4}=\xi^{2}, h_{5}=\eta^{2}, h_{6}=\zeta^{2}, h_{7}=\xi \eta \zeta, \\ h_{8}=\xi^{2} \eta, h_{9}=\xi \zeta, h_{10}=\eta^{2} \xi, h_{11}=\eta^{2} \zeta, h_{12}=\xi \zeta^{2}, h_{13}=\eta \zeta^{2} \\ h_{14}=\xi^{2} \eta \zeta, h_{15}=\xi \eta^{2} \zeta, h_{16}=\xi \eta \zeta^{2}\end{array}\right.$

By evaluating Eq. (6) at the fifteen nodes of the SHB15 element, respectively, at the twenty nodes of the SHB20 element, one obtains the following fifteen-equation system, respectively, twenty-equation system:

$\mathbf{d}_{i}=a_{0 i} \mathbf{s}+a_{1 i} \mathbf{x}_{1}+a_{2 i} \mathbf{x}_{2}+a_{3 i} \mathbf{x}_{3}+c_{1 i} \mathbf{h}_{1}+c_{2 i} \mathbf{h}_{2}+\cdots+c_{\alpha i} \mathbf{h}_{\alpha}, \quad i=1,2,3$, 
where $\mathbf{d}_{i}^{T}=\left(d_{i 1}, d_{i 2}, d_{i 3}, \cdots, d_{i n}\right) \quad$ represent the nodal displacement vectors, and $\mathbf{x}_{i}^{T}=\left(x_{i 1}, x_{i 2}, x_{i 3}, \cdots, x_{i n}\right)$ are the nodal coordinate vectors. The constant vector $\mathbf{s}^{T}=(1,1, \cdots, 1)$ is a fifteen-component vector, in the case of the SHB15 element, and a twenty-component vector, for the SHB20 element. As to vectors $\mathbf{h}_{\alpha}$, these are constant vectors whose expressions can be easily obtained by evaluating the $h_{\alpha}$ functions at the element nodes in the reference coordinate system $(\xi, \eta, \zeta)$ (for the full details, see Abed-Meraim et al. [21]).

By introducing the Hallquist [23] vectors $\mathbf{b}_{i}=\frac{\partial \mathbf{N}}{\partial x_{i}}$, with $\mathbf{N}$ the vector whose components are the shape functions $N_{I}$, one can demonstrate the following first set of orthogonality conditions, which is common to both elements:

$\left\{\begin{array}{l}\mathbf{b}_{i}^{T} \cdot \mathbf{h}_{\alpha}=0 \\ \mathbf{b}_{i}^{T} \cdot \mathbf{s}=0 \\ \mathbf{b}_{i}^{T} \cdot \mathbf{x}_{j}=\delta_{i j}\end{array}\right.$,

where $i, j=1,2,3$, while $\alpha=1, \ldots, 11$, for the SHB15 element, and $\alpha=1, \ldots, 16$, for the SHB20 element.

Then, a second set of orthogonality conditions are established for the SHB15 element

$$
\left\{\begin{array}{l}
\mathbf{h}_{1}^{T} \cdot \mathbf{s}=0, \mathbf{h}_{9}^{T} \cdot \mathbf{s}=0 \\
\mathbf{h}_{2}^{T} \cdot \mathbf{s}=0, \mathbf{h}_{10}^{T} \cdot \mathbf{s}=4 \\
\mathbf{h}_{3}^{T} \cdot \mathbf{s}=\frac{1}{2}, \mathbf{h}_{11}^{T} \cdot \mathbf{s}=4 \\
\mathbf{h}_{4}^{T} \cdot \mathbf{s}=0 \\
\mathbf{h}_{5}^{T} \cdot \mathbf{s}=4 \\
\mathbf{h}_{6}^{T} \cdot \mathbf{s}=4 \\
\mathbf{h}_{7}^{T} \cdot \mathbf{s}=12 \\
\mathbf{h}_{8}^{T} \cdot \mathbf{s}=0
\end{array}\right.
$$

while for the SHB20 element, one obtains 


$$
\left\{\begin{array}{l}
\mathbf{h}_{1}^{T} \cdot \mathbf{s}=0, \mathbf{h}_{9}^{T} \cdot \mathbf{s}=0 \\
\mathbf{h}_{2}^{T} \cdot \mathbf{s}=0, \mathbf{h}_{10}^{T} \cdot \mathbf{s}=0 \\
\mathbf{h}_{3}^{T} \cdot \mathbf{s}=0, \mathbf{h}_{11}^{T} \cdot \mathbf{s}=0 \\
\mathbf{h}_{4}^{T} \cdot \mathbf{s}=16, \mathbf{h}_{12}^{T} \cdot \mathbf{s}=0 \\
\mathbf{h}_{5}^{T} \cdot \mathbf{s}=16, \mathbf{h}_{13}^{T} \cdot \mathbf{s}=0 \\
\mathbf{h}_{6}^{T} \cdot \mathbf{s}=16, \mathbf{h}_{14}^{T} \cdot \mathbf{s}=0 \\
\mathbf{h}_{7}^{T} \cdot \mathbf{s}=0, \mathbf{h}_{15}^{T} \cdot \mathbf{s}=0 \\
\mathbf{h}_{8}^{T} \cdot \mathbf{s}=0, \mathbf{h}_{16}^{T} \cdot \mathbf{s}=0
\end{array} .\right.
$$

Using the above orthogonality conditions (Eqs. (10-12)), and the scalar product of Eq. (9) by $\mathbf{b}_{j}^{T}, \mathbf{s}^{T}$ and $\mathbf{h}_{\alpha}^{T}$, successively, the expression of the unknown constants $a_{j i}$ and $c_{o i}$ in Eqs. (6) and (9) can be obtained as follows:

$a_{j i}=\mathbf{b}_{j}^{T} \cdot \mathbf{d}_{i}, \quad c_{\alpha i}=\gamma_{\alpha}^{T} \cdot \mathbf{d}_{i}$,

where the expressions of vectors $\gamma_{\alpha}$ for the SHB15 element are given by

$$
\begin{aligned}
& \boldsymbol{\gamma}_{\alpha}^{T}=L_{\alpha 1}\left(\mathbf{h}_{1}^{T}-\left(\mathbf{h}_{1}^{T} \cdot \mathbf{x}_{j}\right) \mathbf{b}_{j}^{T}\right)+L_{\alpha 2}\left(\mathbf{h}_{2}^{T}-\left(\mathbf{h}_{2}^{T} \cdot \mathbf{x}_{j}\right) \mathbf{b}_{j}^{T}\right) \\
& +L_{\alpha 3}\left[\left(\mathbf{h}_{3}^{T}-\frac{1}{30} \mathbf{s}^{T}\right)-\left(\left(\mathbf{h}_{3}^{T}-\frac{1}{30} \mathbf{s}^{T}\right) \cdot \mathbf{x}_{j}\right) \mathbf{b}_{j}^{T}\right]+L_{\alpha 4}\left(\mathbf{h}_{4}^{T}-\left(\mathbf{h}_{4}^{T} \cdot \mathbf{x}_{j}\right) \mathbf{b}_{j}^{T}\right) \\
& +L_{\alpha 5}\left[\left(\mathbf{h}_{5}^{T}-\frac{4}{15} \mathbf{s}^{T}\right)-\left(\left(\mathbf{h}_{5}^{T}-\frac{4}{15} \mathbf{s}^{T}\right) \cdot \mathbf{x}_{j}\right) \mathbf{b}_{j}^{T}\right]+L_{\alpha 6}\left[\left(\mathbf{h}_{6}^{T}-\frac{4}{15} \mathbf{s}^{T}\right)-\left(\left(\mathbf{h}_{6}^{T}-\frac{4}{15} \mathbf{s}^{T}\right) \cdot \mathbf{x}_{j}\right) \mathbf{b}_{j}^{T}\right] \\
& +L_{\alpha 7}\left[\left(\mathbf{h}_{7}^{T}-\frac{4}{5} \mathbf{s}^{T}\right)-\left(\left(\mathbf{h}_{7}^{T}-\frac{4}{5} \mathbf{s}^{T}\right) \cdot \mathbf{x}_{j}\right) \mathbf{b}_{j}^{T}\right]+L_{\alpha 8}\left(\mathbf{h}_{8}^{T}-\left(\mathbf{h}_{8}^{T} \cdot \mathbf{x}_{j}\right) \mathbf{b}_{j}^{T}\right) \\
& +L_{\alpha 9}\left(\mathbf{h}_{9}^{T}-\left(\mathbf{h}_{9}^{T} \cdot \mathbf{x}_{j}\right) \mathbf{b}_{j}^{T}\right)+L_{\alpha 10}\left[\left(\mathbf{h}_{10}^{T}-\frac{4}{15} \mathbf{s}^{T}\right)-\left(\left(\mathbf{h}_{10}^{T}-\frac{4}{15} \mathbf{s}^{T}\right) \cdot \mathbf{x}_{j}\right) \mathbf{b}_{j}^{T}\right] \\
& +L_{\alpha 11}\left[\left(\mathbf{h}_{11}^{T}-\frac{4}{15} \mathbf{s}^{T}\right)-\left(\left(\mathbf{h}_{11}^{T}-\frac{4}{15} \mathbf{s}^{T}\right) \cdot \mathbf{x}_{j}\right) \mathbf{b}_{j}^{T}\right]
\end{aligned}
$$

with 


$$
\mathbf{L}_{\alpha \beta}=\left[\begin{array}{ccccccccccc}
\frac{17}{2} & 0 & 0 & -8 & 0 & 0 & 0 & -9 & 0 & 0 & 0 \\
0 & \frac{17}{2} & 0 & -8 & 0 & 0 & 0 & 0 & -9 & 0 & 0 \\
0 & 0 & \frac{256}{17} & 0 & \frac{36}{17} & \frac{36}{17} & 2 & 0 & 0 & -\frac{58}{17} & -\frac{58}{17} \\
-8 & -8 & 0 & 24 & 0 & 0 & 0 & 8 & 8 & 0 & 0 \\
0 & 0 & \frac{36}{17} & 0 & \frac{316}{187} & \frac{146}{187} & 1 & 0 & 0 & -\frac{324}{187} & -\frac{171}{187} \\
0 & 0 & \frac{36}{17} & 0 & \frac{146}{187} & \frac{316}{187} & 1 & 0 & 0 & -\frac{171}{187} & -\frac{324}{187} \\
0 & 0 & 2 & 0 & 1 & 1 & \frac{3}{2} & 0 & 0 & -\frac{3}{2} & -\frac{3}{2} \\
-9 & 0 & 0 & 8 & 0 & 0 & 0 & 10 & 0 & 0 & 0 \\
0 & -9 & 0 & 8 & 0 & 0 & 0 & 0 & 10 & 0 & 0 \\
0 & 0 & -\frac{58}{17} & 0 & -\frac{324}{187} & -\frac{171}{187} & -\frac{3}{2} & 0 & 0 & \frac{505}{187} & \frac{585}{374} \\
0 & 0 & -\frac{58}{17} & 0 & -\frac{171}{187} & -\frac{324}{187} & -\frac{3}{2} & 0 & 0 & \frac{585}{374} & \frac{505}{187}
\end{array}\right]
$$

while for the SHB20 element, vectors $\gamma_{\alpha}$ are given by

$$
\begin{aligned}
& \boldsymbol{\gamma}_{\alpha}^{T}=L_{\alpha 1}\left(\mathbf{h}_{1}^{T}-\left(\mathbf{h}_{1}^{T} \cdot \mathbf{x}_{j}\right) \mathbf{b}_{j}^{T}\right)+L_{\alpha 2}\left(\mathbf{h}_{2}^{T}-\left(\mathbf{h}_{2}^{T} \cdot \mathbf{x}_{j}\right) \mathbf{b}_{j}^{T}\right) \\
& +L_{\alpha 3}\left(\mathbf{h}_{3}^{T}-\left(\mathbf{h}_{3}^{T} \cdot \mathbf{x}_{j}\right) \mathbf{b}_{j}^{T}\right)+L_{\alpha 4}\left[\left(\mathbf{h}_{4}^{T}-\frac{4}{5} \mathbf{s}^{T}\right)-\left(\left(\mathbf{h}_{4}^{T}-\frac{4}{5} \mathbf{s}^{T}\right) \cdot \mathbf{x}_{j}\right) \mathbf{b}_{j}^{T}\right] \\
& \quad+L_{\alpha 5}\left[\left(\mathbf{h}_{5}^{T}-\frac{4}{5} \mathbf{s}^{T}\right)-\left(\left(\mathbf{h}_{5}^{T}-\frac{4}{5} \mathbf{s}^{T}\right) \cdot \mathbf{x}_{j}\right) \mathbf{b}_{j}^{T}\right]+L_{\alpha 6}\left[\left(\mathbf{h}_{6}^{T}-\frac{4}{5} \mathbf{s}^{T}\right)-\left(\left(\mathbf{h}_{6}^{T}-\frac{4}{5} \mathbf{s}^{T}\right) \cdot \mathbf{x}_{j}\right) \mathbf{b}_{j}^{T}\right] \\
& \quad+L_{\alpha 7}\left(\mathbf{h}_{7}^{T}-\left(\mathbf{h}_{7}^{T} \cdot \mathbf{x}_{j}\right) \mathbf{b}_{j}^{T}\right)+L_{\alpha 8}\left(\mathbf{h}_{8}^{T}-\left(\mathbf{h}_{8}^{T} \cdot \mathbf{x}_{j}\right) \mathbf{b}_{j}^{T}\right) \\
& +L_{\alpha 9}\left(\mathbf{h}_{9}^{T}-\left(\mathbf{h}_{9}^{T} \cdot \mathbf{x}_{j}\right) \mathbf{b}_{j}^{T}\right)+L_{\alpha 10}\left(\mathbf{h}_{10}^{T}-\left(\mathbf{h}_{10}^{T} \cdot \mathbf{x}_{j}\right) \mathbf{b}_{j}^{T}\right) \\
& +L_{\alpha 11}\left(\mathbf{h}_{11}^{T}-\left(\mathbf{h}_{11}^{T} \cdot \mathbf{x}_{j}\right) \mathbf{b}_{j}^{T}\right)+L_{\alpha 12}\left(\mathbf{h}_{12}^{T}-\left(\mathbf{h}_{12}^{T} \cdot \mathbf{x}_{j}\right) \mathbf{b}_{j}^{T}\right) \\
& +L_{\alpha 13}\left(\mathbf{h}_{13}^{T}-\left(\mathbf{h}_{13}^{T} \cdot \mathbf{x}_{j}\right) \mathbf{b}_{j}^{T}\right)+L_{\alpha 14}\left(\mathbf{h}_{14}^{T}-\left(\mathbf{h}_{14}^{T} \cdot \mathbf{x}_{j}\right) \mathbf{b}_{j}^{T}\right) \\
& +L_{\alpha 15}\left(\mathbf{h}_{15}^{T}-\left(\mathbf{h}_{15}^{T} \cdot \mathbf{x}_{j}\right) \mathbf{b}_{j}^{T}\right)+L_{\alpha 16}\left(\mathbf{h}_{16}^{T}-\left(\mathbf{h}_{16}^{T} \cdot \mathbf{x}_{j}\right) \mathbf{b}_{j}^{T}\right)
\end{aligned}
$$


with

$$
\mathbf{L}_{\alpha \beta}=\left[\begin{array}{cccccccccccccccc}
\frac{1}{4} & 0 & 0 & 0 & 0 & 0 & 0 & 0 & 0 & 0 & 0 & 0 & 0 & 0 & -\frac{1}{4} & 0 \\
0 & \frac{1}{4} & 0 & 0 & 0 & 0 & 0 & 0 & 0 & 0 & 0 & 0 & 0 & -\frac{1}{4} & 0 & 0 \\
0 & 0 & \frac{1}{4} & 0 & 0 & 0 & 0 & 0 & 0 & 0 & 0 & 0 & 0 & 0 & 0 & -\frac{1}{4} \\
0 & 0 & 0 & \frac{3}{8} & \frac{1}{8} & \frac{1}{8} & 0 & 0 & 0 & 0 & 0 & 0 & 0 & 0 & 0 & 0 \\
0 & 0 & 0 & \frac{1}{8} & \frac{3}{8} & \frac{1}{8} & 0 & 0 & 0 & 0 & 0 & 0 & 0 & 0 & 0 & 0 \\
0 & 0 & 0 & \frac{1}{8} & \frac{1}{8} & \frac{3}{8} & 0 & 0 & 0 & 0 & 0 & 0 & 0 & 0 & 0 & 0 \\
0 & 0 & 0 & 0 & 0 & 0 & \frac{1}{8} & 0 & 0 & 0 & 0 & 0 & 0 & 0 & 0 & 0 \\
0 & 0 & 0 & 0 & 0 & 0 & 0 & \frac{3}{20} & 0 & 0 & 0 & 0 & -\frac{1}{10} & 0 & 0 & 0 \\
0 & 0 & 0 & 0 & 0 & 0 & 0 & 0 & 0 & \frac{3}{20} & 0 & -\frac{1}{10} & 0 & 0 & 0 & 0 \\
0 & 0 & 0 & 0 & 0 & 0 & 0 & 0 & -\frac{1}{10} & 0 & \frac{3}{20} & 0 & 0 & 0 & 0 & 0 \\
0 & 0 & 0 & 0 & 0 & 0 & 0 & 0 & 0 & -\frac{1}{10} & 0 & \frac{3}{20} & 0 & 0 & 0 & 0 \\
0 & 0 & 0 & 0 & 0 & 0 & 0 & -\frac{1}{10} & 0 & 0 & 0 & 0 & \frac{3}{20} & 0 & 0 & 0 \\
0 & 0 & 0 & 0 & 0 & 0 & 0 & 0 & 0 & 0 & 0 & 0 & \frac{1}{8} & 0 & 0 \\
0 & 0 & 0 & 0 & 0 & 0 & 0 & 0 & 0 & 0 & 0 & 0 & 0 & 0 & \frac{1}{8}
\end{array}\right]
$$

By differentiating Eq. (6) and using Eq. (13), the expression of the displacement gradient $u_{i, j}$ is derived as follows:

$$
u_{i, j}=\left(\mathbf{b}_{j}^{T}+h_{\alpha, j} \gamma_{\alpha}^{T}\right) \cdot \mathbf{d}_{i}
$$


with $\alpha$ varying from 1 to 11 for the SHB15 element, and from 1 to 16 for the SHB20 element.

Finally, the expression of the strain field, which is related to the nodal displacements by the discrete gradient operator $\mathbf{B}$, is given by

$$
\nabla_{s}(\mathbf{u})=\left[\begin{array}{c}
u_{x, x} \\
u_{y, y} \\
u_{z, z} \\
u_{x, y}+u_{y, x} \\
u_{y, z}+u_{z, y} \\
u_{x, z}+u_{z, x}
\end{array}\right]=\mathbf{B} \cdot \mathbf{d}=\mathbf{B} \cdot\left[\begin{array}{c}
\mathbf{d}_{x} \\
\mathbf{d}_{y} \\
\mathbf{d}_{z}
\end{array}\right],
$$

where the discrete gradient operator $\mathbf{B}$ takes the following matrix form:

$$
\mathbf{B}=\left[\begin{array}{ccc}
\mathbf{b}_{x}^{T}+h_{\alpha, x} \boldsymbol{\gamma}_{\alpha}^{T} & \mathbf{0} & \mathbf{0} \\
\mathbf{0} & \mathbf{b}_{y}^{T}+h_{\alpha, y} \boldsymbol{\gamma}_{\alpha}^{T} & \mathbf{0} \\
\mathbf{0} & \mathbf{0} & \mathbf{b}_{z}^{T}+h_{\alpha, z} \boldsymbol{\gamma}_{\alpha}^{T} \\
\mathbf{b}_{y}^{T}+h_{\alpha, y} \boldsymbol{\gamma}_{\alpha}^{T} & \mathbf{b}_{x}^{T}+h_{\alpha, x} \gamma_{\alpha}^{T} & \mathbf{0} \\
\mathbf{0} & \mathbf{b}_{z}^{T}+h_{\alpha, z} \boldsymbol{\gamma}_{\alpha}^{T} & \mathbf{b}_{y}^{T}+h_{\alpha, y} \boldsymbol{\gamma}_{\alpha}^{T} \\
\mathbf{b}_{z}^{T}+h_{\alpha, z} \boldsymbol{\gamma}_{\alpha}^{T} & \mathbf{0} & \mathbf{b}_{x}^{T}+h_{\alpha, x} \boldsymbol{\gamma}_{\alpha}^{T}
\end{array}\right] .
$$

\subsection{Variational principle}

The assumed-strain formulation of the SHB15 and SHB20 solid-shell elements is based on the simplified form of the $\mathrm{Hu}-$ Washizu mixed variational principle, as suggested by Simo and Hughes [24], which writes at the element level

$$
\delta \pi(\dot{\overline{\boldsymbol{\varepsilon}}})=\int_{\Omega_{e}} \delta \dot{\overline{\boldsymbol{\varepsilon}}}^{T} \cdot \boldsymbol{\sigma} d \Omega-\delta \dot{\mathbf{d}}^{T} \cdot \mathbf{f}^{e x t}=0,
$$

where $\delta$ denotes a variation, $\dot{\overline{\boldsymbol{\varepsilon}}}$ the assumed-strain rate, $\boldsymbol{\sigma}$ the Cauchy stress tensor, $\dot{\mathbf{d}}$ the nodal velocities, and $\mathbf{f}^{\text {ext }}$ the external nodal forces. It is worth noting that in the formulation of the linear versions of the solid-shell elements (i.e., SHB6 and SHB8PS, see, e.g., Abed-Meraim and Combescure [12], Trinh et al. [20]), the assumed-strain rate $\dot{\bar{\varepsilon}}$ has been expressed in terms of a projected matrix $\overline{\mathbf{B}}$, which is derived from the classical $\mathbf{B}$ operator, in order to eliminate most locking phenomena (e.g., membrane locking, shear locking, etc.). For the present quadratic solid-shell elements (SHB15 and SHB20), no significant locking has been revealed when 
evaluating their performance on a selective and representative set of benchmark problems (see Abed-Meraim et al. [21]). Consequently, no projection is applied to the discrete gradient operator $\mathbf{B}$ and, accordingly, the expression of the assumed-strain rate reduces to

$\dot{\overline{\boldsymbol{\varepsilon}}}(x, t)=\mathbf{B} \cdot \dot{\mathbf{d}}$.

Substituting the above equation into the simplified form of the $\mathrm{Hu}-$ Washizu variational principle, the expressions of the element stiffness matrix and internal force vector are obtained as follows:

$\mathbf{K}_{e}=\int_{\Omega_{e}} \mathbf{B}^{T} \cdot \mathbf{C}^{\mathrm{ep}} \cdot \mathbf{B} d \Omega, \quad \mathbf{f}^{\mathrm{int}}=\int_{\Omega_{e}} \mathbf{B}^{T} \cdot \boldsymbol{\sigma}(\dot{\overline{\boldsymbol{\varepsilon}}}) d \Omega$,

where $\mathbf{C}^{\mathrm{ep}}$ is the fourth-order elasto-plastic tangent modulus, whose expression will be detailed in the following subsection.

\subsection{Constitutive equations}

The formulation of the quadratic solid-shell elements SHB15 and SHB20 is extended in this paper to the framework of large displacements and rotations, and is coupled with advanced largestrain anisotropic constitutive equations for metallic materials. In this process, two types of local frames need to be introduced with respect to the global coordinate system, as illustrated in Fig. 3 . The first type of local frame, which has already been defined in Section 2.1 (see Fig. 2) and denoted as the "element frame", is attached to the element mid-plane associated with each integration point. The second type of local physical coordinate system is the so-called "material frame", which is introduced to define the anisotropic plastic behavior of the material. The time integration of the large-strain anisotropic elasto-plastic constitutive equations, which is achieved at each integration point, also uses this local material frame in order to satisfy the objectivity (material invariance) requirements. Both the local element frame and the material frame are defined relative to the global coordinate frame by their rotation matrix $\mathbf{P}$ and $\mathscr{R}$, respectively, which allows mapping any vector $\mathbf{a}$ or tensor $\mathbf{A}$ from local to global coordinate systems. 


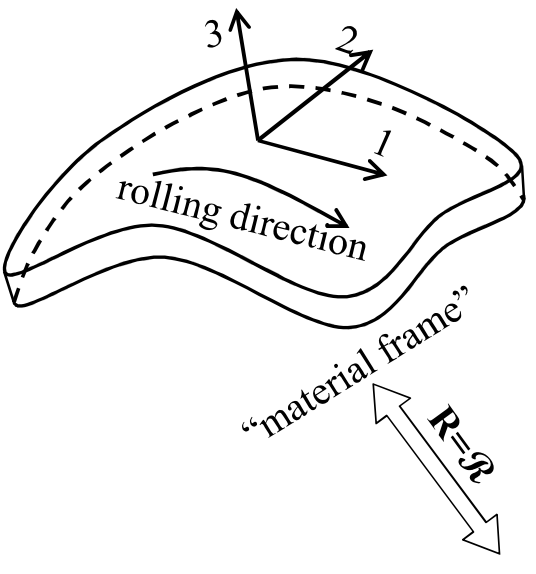

$\zeta$ thickness direction

integration points

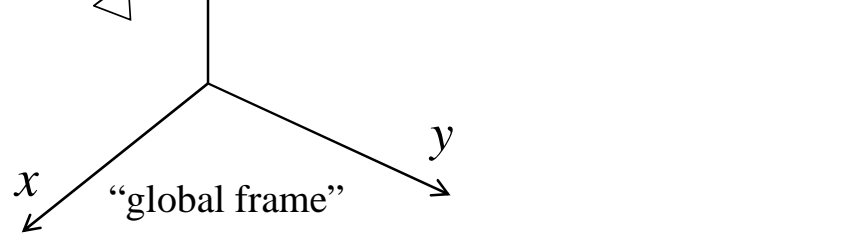

Fig. 3. Illustration of the local coordinate systems used in the formulation of the quadratic solid-shell elements.

For the modeling of the anisotropic plastic behavior, the quadratic Hill'48 yield criterion [25] is coupled with the formulation of the SHB elements. Accordingly, the plastic yield function is given by

$F=\sqrt{\left(\boldsymbol{\sigma}^{\prime}-\boldsymbol{\alpha}\right): \mathbf{M}:\left(\boldsymbol{\sigma}^{\prime}-\boldsymbol{\alpha}\right)}-Y$,

where $\boldsymbol{\sigma}^{\prime}$ denotes the deviatoric part of the Cauchy stress tensor $\boldsymbol{\sigma}$, and $\boldsymbol{\alpha}$ is the back-stress tensor, which describes the kinematic hardening of the material. The fourth-order tensor $\mathbf{M}$ contains the Hill anisotropy coefficients $(\mathrm{F}, \mathrm{G}, \mathrm{H}, \mathrm{L}, \mathrm{M}$ and $\mathrm{N})$. The isotropic hardening of the material, which characterizes the size of the yield surface, is modeled by the scalar function $Y$.

The plastic strain rate tensor $\mathbf{D}^{\mathrm{p}}$ is obtained using the classical associative plastic flow rule, which follows the normality law with respect to the yield surface

$\mathbf{D}^{\mathrm{p}}=\dot{\lambda} \frac{\partial F}{\partial \boldsymbol{\sigma}}=\dot{\lambda} \mathbf{V}$

where $\dot{\lambda}$ and $\mathbf{V}$ represent the plastic multiplier and the plastic flow direction, respectively. 
In the local material frame, the Cauchy stress rate can be expressed using the following hypoelastic law:

$\dot{\boldsymbol{\sigma}}=\mathbf{C}^{\mathrm{e}}:\left(\mathbf{D}-\mathbf{D}^{\mathrm{p}}\right)$

where the second-order tensor $\mathbf{D}$ denotes the total strain rate, while $\mathbf{C}^{\mathrm{e}}$ is the fourth-order elasticity tensor. Note that a modified plane-stress-type elasticity matrix has been adopted in the formulation of the linear versions of the SHB elements (see, e.g., Abed-Meraim and Combescure [12], Trinh et al. [20]), in order to avoid the locking phenomena encountered when the classical fully three-dimensional elasticity tensor is considered. By contrast, such a modification is not required for the present quadratic versions of the SHB elements, since their performance has been assessed with both the plane-stress-type elasticity matrix and the classical fully three-dimensional one, showing quite equivalent results. Therefore, the classical fully three-dimensional elasticity matrix is implemented with the proposed quadratic versions of the SHB elements, which represents a major advantage with respect to their linear counterparts.

The plastic multiplier $\dot{\lambda}$ in Eq. (21) is determined by using the consistency condition $\dot{F}=0$, which leads to

$\dot{\lambda}=\frac{\mathbf{V}: \mathbf{C}^{\mathrm{e}}: \mathbf{D}}{\mathbf{V}: \mathbf{C}^{\mathrm{e}}: \mathbf{V}+\mathbf{V}: \mathbf{H}_{\boldsymbol{a}}+H_{Y}}$

where the hardening moduli $H_{Y}$ and $\mathbf{H}_{\boldsymbol{\alpha}}$ are scalar and tensor components involved in the evolution laws describing the isotropic and kinematic hardening, respectively. The latter can be expressed in the following generic form:

$\left\{\begin{array}{l}\dot{Y}=H_{Y} \dot{\lambda} \\ \dot{\boldsymbol{\alpha}}=\mathbf{H}_{\boldsymbol{\alpha}} \dot{\lambda}\end{array}\right.$.

Finally, by substituting the expression of the plastic multiplier $\dot{\lambda}$ into the hypo-elastic law (22), the elasto-plastic tangent modulus is derived as

$$
\mathbf{C}^{\mathrm{ep}}=\mathbf{C}^{\mathrm{e}}-\gamma \frac{\left(\mathbf{C}^{\mathrm{e}}: \mathbf{V}\right) \otimes\left(\mathbf{V}: \mathbf{C}^{\mathrm{e}}\right)}{\mathbf{V}: \mathbf{C}^{\mathrm{e}}: \mathbf{V}+\mathbf{V}: \mathbf{H}_{\boldsymbol{\alpha}}+H_{Y}}
$$

where $\gamma=0$ for elastic loading/unloading, and $\gamma=1$ for strict plastic loading. 


\section{Simulation of linear and nonlinear benchmark problems}

The formulations of the quadratic solid-shell elements (SHB15 and SHB20) presented above have been implemented into the finite element code ABAQUS/Standard. A representative set of linear and nonlinear benchmark tests is selected in this section to evaluate the performance of the proposed SHB elements. The obtained results are systematically compared, on the one hand, with those provided by ABAQUS quadratic elements, using the same in-plane meshes, and on the other hand with reference solutions taken from the literature. The description of the finite elements used for comparison purposes is given in Table 1. Note that, for the proposed SHB15 and SHB20 formulations, only two integration points along the thickness are sufficient to model the following linear and nonlinear elastic benchmark tests, while three integration points are used in the case of elasto-plastic benchmark problems.

In this section, all geometries are discretized using the following nomenclature. For hexahedral elements, meshes of $\mathrm{N}_{1} \times \mathrm{N}_{2} \times \mathrm{N}_{3}$ elements are adopted, where $\mathrm{N}_{1}$ denotes the number of elements in the length direction, $\mathrm{N}_{2}$ is the number of elements in the width direction, and $\mathrm{N}_{3}$ is the number of elements in the thickness direction. For meshes with prismatic elements, the nomenclature adopted is $\left(\mathrm{N}_{1} \times \mathrm{N}_{2} \times 2\right) \times \mathrm{N}_{3}$, which corresponds to twice the total number of elements involved in hexahedron-based meshes, due to the subdivision of each hexahedron into two prisms. For ABAQUS shell elements, the nomenclature adopted for quadrilateral shell elements is $\mathrm{N}_{1} \times \mathrm{N}_{2}$, while the nomenclature for triangular shell elements is $\mathrm{N}_{1} \times \mathrm{N}_{2} \times 2$. 
Table 1

Quadratic prismatic, hexahedral, and shell finite elements used in the simulations.

\begin{tabular}{|c|c|c|}
\hline \multirow{3}{*}{$\begin{array}{l}\text { Prismatic elements / } \\
\text { Triangular shell element }\end{array}$} & SHB15 & $\begin{array}{l}\text { 15-node prismatic solid-shell element with a user- } \\
\text { defined number of through-thickness integration points }\end{array}$ \\
\hline & C3D15 & $\begin{array}{l}\text { 15-node prismatic solid element with three integration } \\
\text { points through the thickness }\end{array}$ \\
\hline & STRI65 & $\begin{array}{l}\text { 6-node triangular shell element with a user-defined } \\
\text { number of through-thickness integration points }\end{array}$ \\
\hline \multirow{3}{*}{$\begin{array}{l}\text { Hexahedral elements / } \\
\text { Quadrilateral shell } \\
\text { element }\end{array}$} & SHB20 & $\begin{array}{l}\text { 20-node hexahedral solid-shell element with a user- } \\
\text { defined number of through-thickness integration points }\end{array}$ \\
\hline & C3D20 & $\begin{array}{l}\text { 20-node hexahedral solid element with three } \\
\text { integration points through the thickness }\end{array}$ \\
\hline & S8R & $\begin{array}{l}\text { 8-node reduced-integration quadrilateral shell element } \\
\text { with a user-defined number of through-thickness } \\
\text { integration points }\end{array}$ \\
\hline
\end{tabular}

\subsection{Bending of a clamped square plate}

The performance of the proposed SHB elements is first evaluated on a linear elastic problem, which consists of a clamped square plate subjected to a central concentrated force. The geometric dimensions, material properties, and boundary conditions of the problem are all illustrated in Fig. 4. The value of the concentrated point load is chosen so that the analytical displacement at the center of the plate is $u_{r e f}=0.0056 \frac{\mathrm{FL}^{2}}{D}=1$, where $D=\frac{E \mathrm{t}^{3}}{12\left(1-v^{2}\right)}$ is the flexural rigidity of the plate [26]. Owing to the symmetry, only one quarter of the plate is discretized using three different regular meshes, in order to assess the convergence rate of the proposed SHB elements. The convergence results for the SHB elements, in terms of central point displacement normalized with respect to the analytical displacement $u_{r e f}=1$, are shown in Fig. 5 along with the results given by ABAQUS quadratic elements. Among the prismatic and triangular elements, the ABAQUS quadratic shell element STRI65 has the fastest convergence, followed by the proposed SHB15 element, while the convergence of the ABAQUS quadratic solid element C3D15 is the slowest. For the hexahedral and quadrilateral elements, the convergence of the proposed SHB20 element is similar to that of the ABAQUS quadratic shell element S8R, which is much faster than that of the ABAQUS quadratic solid element C3D20. 


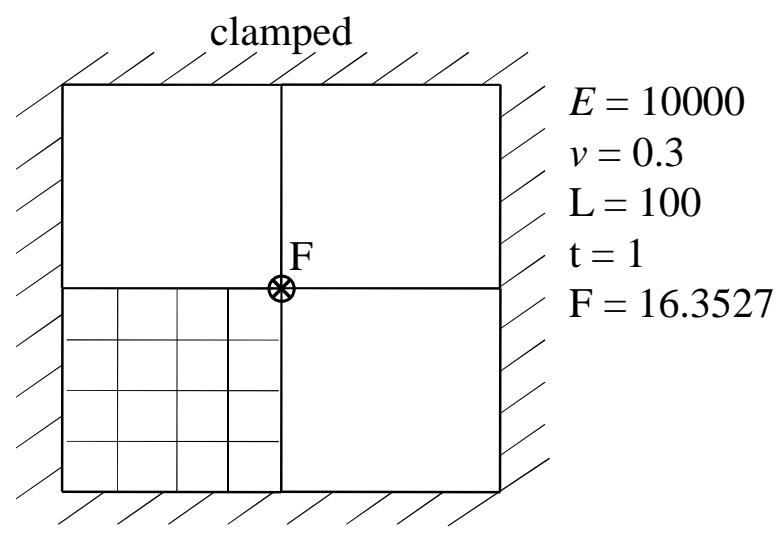

Fig. 4. Geometry, material properties, and boundary conditions for the clamped square plate.

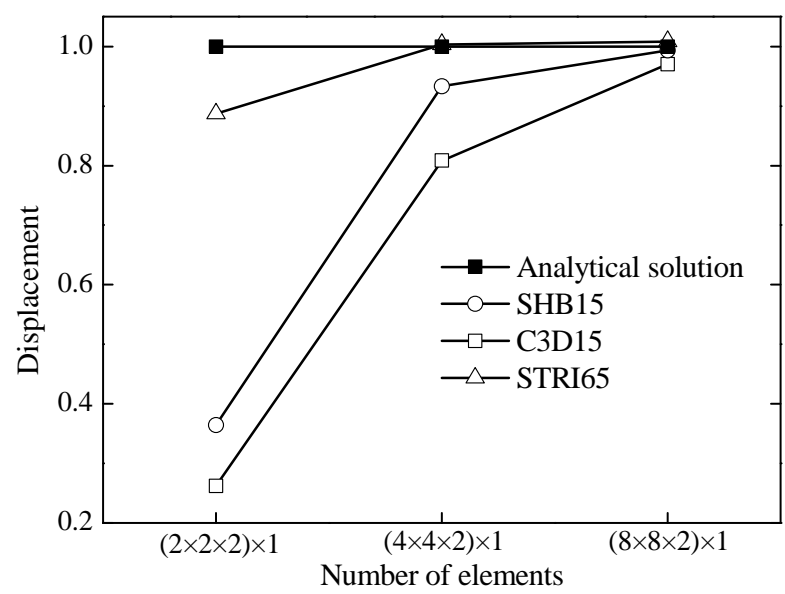

(a) triangular shell / prismatic elements

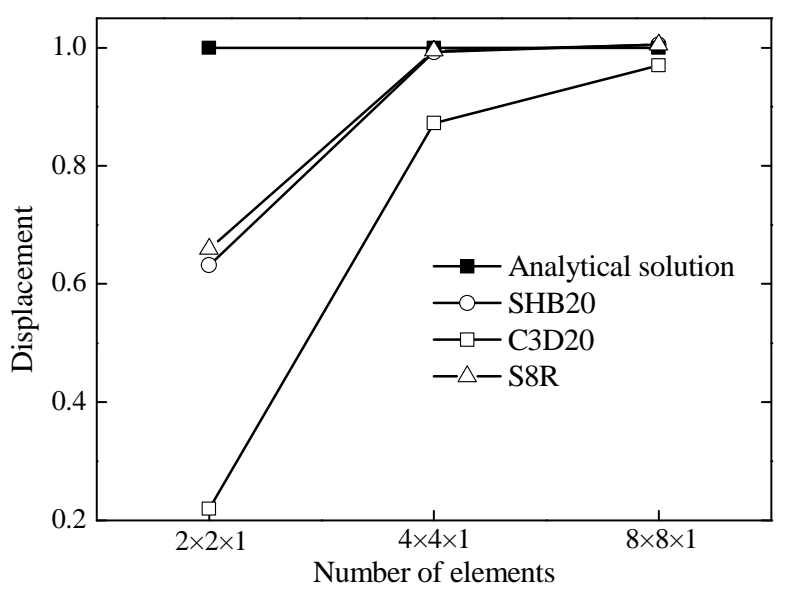

(b) quadrilateral shell / hexahedral elements

Fig. 5. Convergence results for the clamped square plate subjected to a central concentrated force.

In addition to the convergence results above, a sensitivity analysis with respect to the in-plane mesh distortion is conducted here, as proposed by Alves de Sousa et al. [8]. To this end, a quarter of the square plate is discretized by $(2 \times 2 \times 2) \times 1$ elements, in the case of triangular shell or prismatic elements, and by $2 \times 2 \times 1$ elements, in the case of quadrilateral shell or hexahedral elements. The mesh distortion is created by moving the central node of the mesh (see point $B$ in Fig. 6) with a predefined distance $d(0 \leq d \leq 12)$, as illustrated in Fig. 6. Again, the normalized displacement of the central point A (see Fig. 6), as a function of the distortion parameter d, is investigated. Figure 7 shows the effect of the distortion parameter $d$ on the normalized displacement of the central point A, as obtained with the SHB elements and ABAQUS quadratic 
solid and shell elements. With regard to mesh distortion sensitivity, the ABAQUS triangular shell element STRI65 shows better performance than the SHB15 element; nevertheless, the latter performs better than the ABAQUS prismatic solid element C3D15. For the hexahedral elements, the sensitivity of the proposed SHB20 element to mesh distortion is similar to that displayed by the ABAQUS shell element S8R, while the ABAQUS quadratic solid element C3D20 exhibits the highest sensitivity to mesh distortion and provides poor results with respect to the reference solution for all values of the distortion parameter $d$.

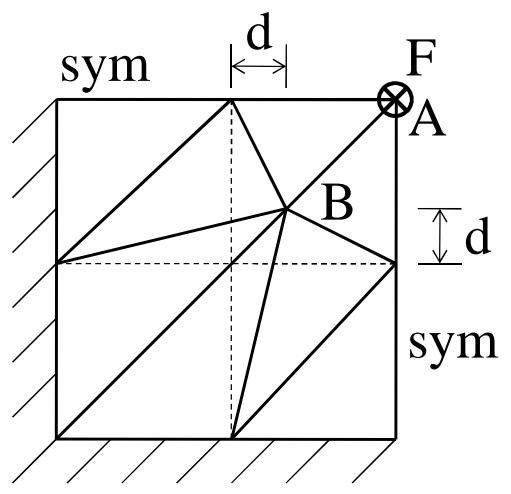

(a) triangular shell / prismatic elements

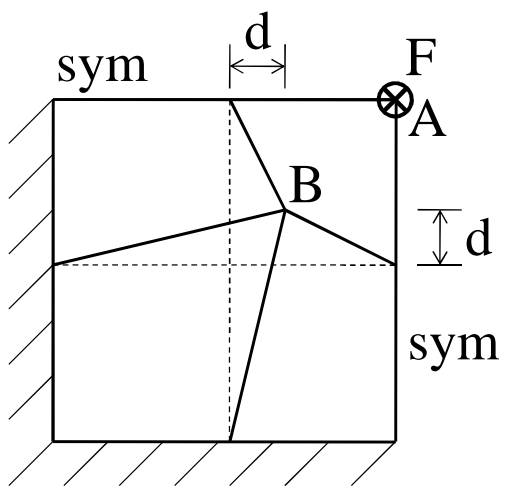

(b) quadrilateral shell / hexahedral elements

Fig. 6. Illustration of in-plane distorted meshes for a quarter of the clamped square plate.

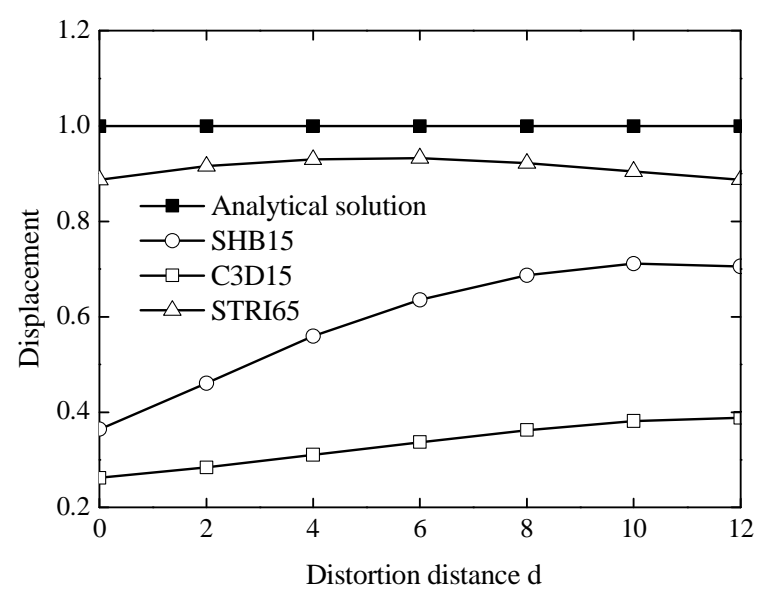

(a) triangular shell / prismatic elements

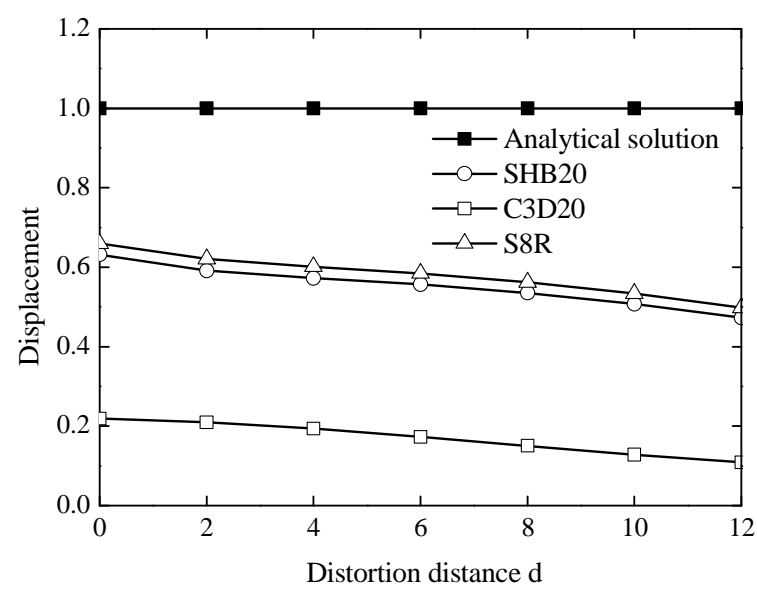

(b) quadrilateral shell / hexahedral elements

Fig. 7. Effect of the in-plane mesh distortion on the normalized displacement of the center point of the clamped square plate. 


\subsection{Bending of a clamped rectangular plate}

The second linear elastic problem consists of a clamped rectangular plate as shown in Fig. 8 . Two types of bending loading are considered: a concentrated force $F=4 \times 10^{-4}$ at the central point of the plate, and a uniform pressure $P=10^{-4}$ at the top surface of the plate. Within the small-strain framework, the deflection at the central point of the plate can be determined analytically by the following expressions, which are taken from reference [26]:

Concentrated force loading: $\mathrm{U}_{Z(\text { ref })}=0.00725 \frac{F b^{2}}{D} \approx 7.23 \times 10^{-6}$

Uniform pressure loading: $\mathrm{U}_{Z(\text { ref })}=0.0026 \frac{P b^{4}}{D} \approx 2.56 \times 10^{-6}$

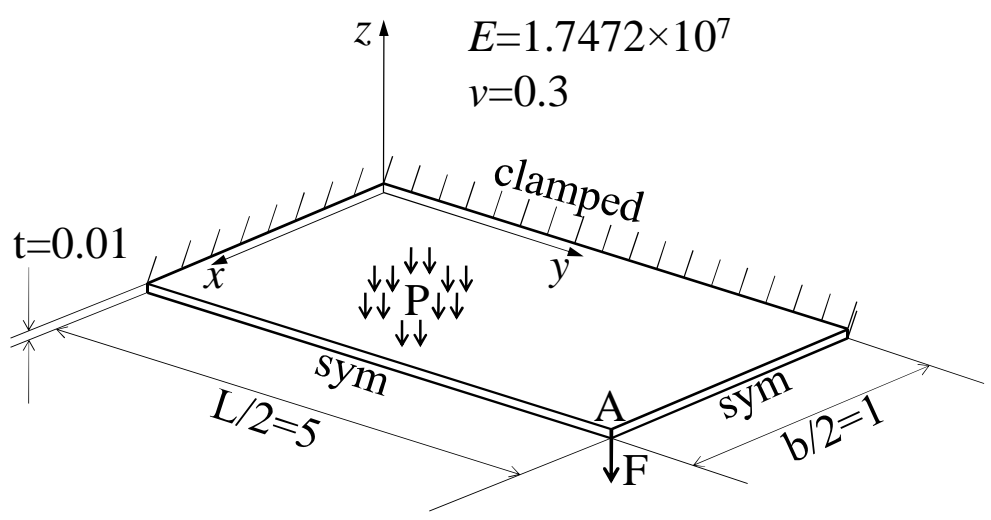

Fig. 8. Geometry, material properties, and boundary conditions for the clamped rectangular plate.

Owing to the symmetry, only one quarter of the plate is analyzed using the SHB15 and SHB20 elements. The convergence results in terms of normalized deflections at the central point of the plate (point A in Fig. 8), corresponding to both types of loading, are reported in Tables 2 and 3. One can observe that, for this linear elastic test problem, the SHB20 solid-shell element has a convergence rate similar to that of the ABAQUS shell element S8R, for both considered types of loading, while the convergence of the ABAQUS solid element C3D20 is much slower. For the SHB15 prismatic solid-shell element, the convergence is slightly slower than that of the ABAQUS triangular shell element STRI65, but faster than that of the ABAQUS prismatic solid element C3D15. 
Table 2

Normalized deflection at point A: case of concentrated force.

\begin{tabular}{|c|c|c|c|c|c|c|c|}
\hline Number of & STRI65 & C3D15 & SHB15 & \multirow{2}{*}{$\begin{array}{l}\text { Number of } \\
\text { elements }\end{array}$} & S8R & C3D20 & SHB20 \\
\hline elements & $\mathrm{U}_{\mathrm{Z}} / \mathrm{U}_{\mathrm{Z}(\mathrm{ref})}$ & $\mathrm{U}_{\mathrm{Z}} / \mathrm{U}_{\mathrm{Z}(\mathrm{ref})}$ & $\mathrm{U}_{\mathrm{Z}} / \mathrm{U}_{\mathrm{Z}(\mathrm{ref})}$ & & $\mathrm{U}_{\mathrm{Z}} / \mathrm{U}_{\mathrm{Z}(\mathrm{ref})}$ & $\mathrm{U}_{\mathrm{Z}} / \mathrm{U}_{\mathrm{Z}(\mathrm{ref})}$ & $\mathrm{U}_{\mathrm{Z}} / \mathrm{U}_{\mathrm{Z}(\mathrm{ref})}$ \\
\hline$(5 \times 1 \times 2) \times 1$ & 0.1834 & 0.0614 & 0.1845 & $5 \times 1 \times 1$ & 0.9526 & 0.0023 & 0.9510 \\
\hline$(10 \times 2 \times 2) \times 1$ & 0.8639 & 0.4618 & 0.6536 & $10 \times 2 \times 1$ & 0.8163 & 0.3956 & 0.8017 \\
\hline$(20 \times 4 \times 2) \times 1$ & 0.9934 & 0.8139 & 0.9089 & $20 \times 4 \times 1$ & 0.9905 & 0.8643 & 0.9890 \\
\hline$(50 \times 10 \times 2) \times 1$ & 1.0037 & 0.9743 & 0.9916 & $50 \times 10 \times 1$ & 1.0019 & 0.9755 & 1.0018 \\
\hline
\end{tabular}

Table 3

Normalized deflection at point A: case of uniform pressure.

\begin{tabular}{|c|c|c|c|c|c|c|c|}
\hline \multirow{2}{*}{$\begin{array}{l}\text { Number of } \\
\text { elements }\end{array}$} & STRI65 & C3D15 & SHB15 & \multirow{2}{*}{$\begin{array}{l}\text { Number of } \\
\text { elements }\end{array}$} & S8R & $\mathrm{C} 3 \mathrm{D} 20$ & SHB20 \\
\hline & $\mathrm{U}_{\mathrm{Z}} / \mathrm{U}_{\mathrm{Z}(\mathrm{ref})}$ & $\mathrm{U}_{\mathrm{Z}} / \mathrm{U}_{\mathrm{Z}(\mathrm{ref})}$ & $\mathrm{U}_{\mathrm{Z}} / \mathrm{U}_{\mathrm{Z}(\mathrm{ref})}$ & & $\mathrm{U}_{\mathrm{Z}} / \mathrm{U}_{\mathrm{Z}(\mathrm{ref})}$ & $\mathrm{U}_{\mathrm{Z}} / \mathrm{U}_{\mathrm{Z}(\mathrm{ref})}$ & $\mathrm{U}_{\mathrm{Z}} / \mathrm{U}_{\mathrm{Z} \text { (ref) }}$ \\
\hline$(5 \times 1 \times 2) \times 1$ & 0.3177 & 0.0149 & 0.0055 & $5 \times 1 \times 1$ & 1.0208 & 0.0018 & 1.0201 \\
\hline$(10 \times 2 \times 2) \times 1$ & 1.0102 & 0.5747 & 0.7762 & $10 \times 2 \times 1$ & 1.0255 & 0.6960 & 1.0289 \\
\hline$(20 \times 4 \times 2) \times 1$ & 1.0248 & 0.9233 & 0.9667 & $20 \times 4 \times 1$ & 1.0176 & 0.9046 & 1.0176 \\
\hline
\end{tabular}

\subsection{Pull-out of an open-ended cylindrical shell}

In this test problem, and some others that follow, the performance of the SHB elements will be evaluated in the framework of geometric nonlinearities (i.e., large displacements and rotations). The first test in this category consists of a free elastic open-ended cylindrical shell, which is pulled out by two opposite radial forces as illustrated in Fig. 9. This benchmark test has been studied by several authors (see, e.g., [14, 27-29]), due to its particular boundary conditions involving very large rotations. Considering the problem symmetry, only one eighth of the cylindrical shell is modeled, as shown in Fig. 9. The load-displacement curves at point A in the 
$z$-direction and at points B and C in the $x$-direction, which are obtained with the SHB elements, are compared in Fig. 10 with those given by ABAQUS elements as well as with the reference solution taken from Sze et al. [29]. The shape of the load-displacement curves reveals that the solution exhibits two main stages: the first stage is governed by bending effects, which is characterized by large displacements and rotations, while the second stage is dominated by membrane effects. The transition between the two stages is marked by a snap-through point at a critical force value of $22 \times 10^{3}$, which is characterized by a reversal of displacement of point $\mathrm{C}$ in the load-displacement curve. The load-displacement curves obtained with the SHB elements are in excellent agreement with the reference solution as well as with those given by ABAQUS elements. However, the C3D20 ABAQUS element requires finer meshes in order to obtain an accurate solution for this severe benchmark test.

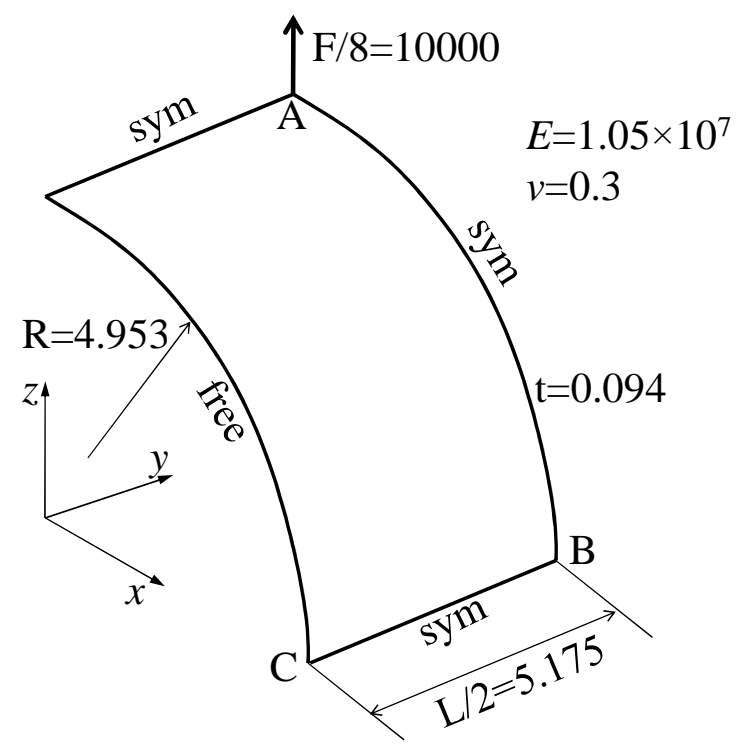

Fig. 9. Geometry, elastic properties, and boundary conditions for the open-ended cylindrical shell subjected to radial pulling forces. 


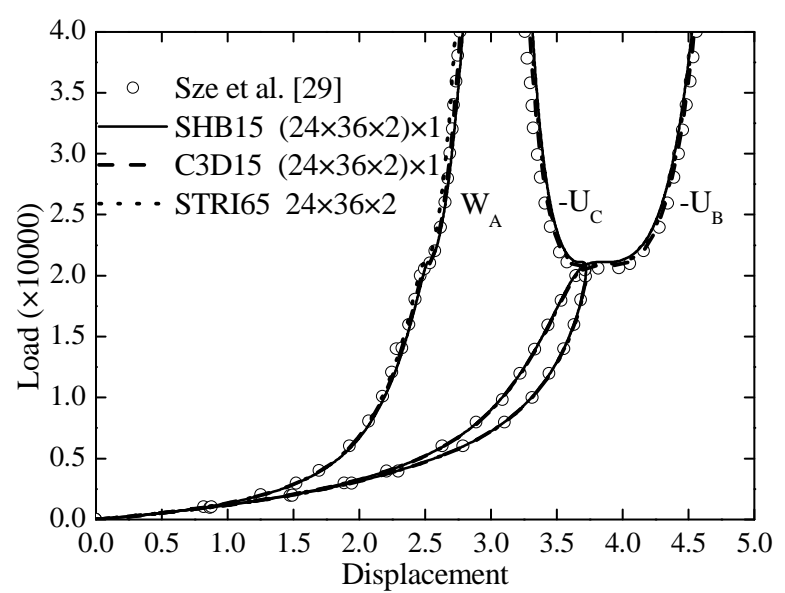

(a) triangular shell / prismatic elements

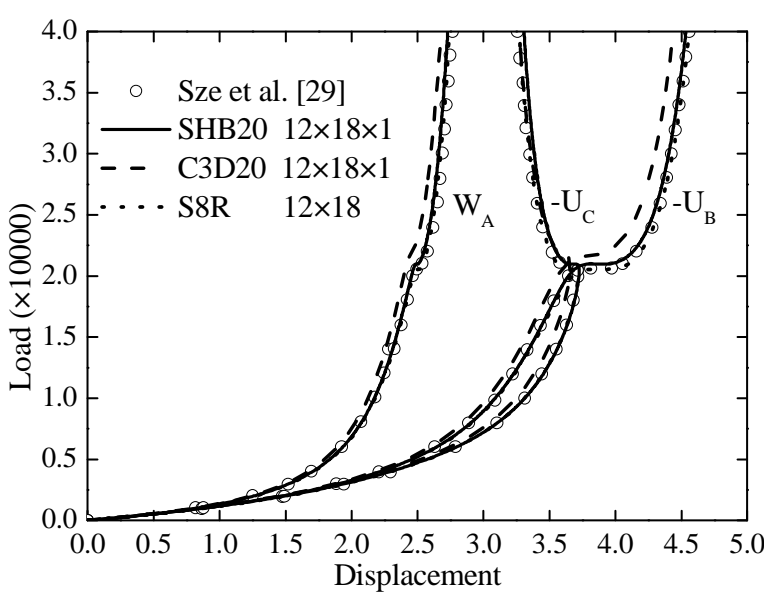

(b) quadrilateral shell / hexahedral elements

Fig. 10. Load-displacement curves for the open-ended cylindrical shell subjected to radial pulling forces.

\subsection{Hemispherical shell with a hole}

Figure 11 illustrates a free hemispherical shell with an $18^{\circ}$ circular hole at its pole (see Park et al. [30], Sze et al. [31]). The shell is loaded by a pair of alternating forces at $90^{\circ}$ intervals. Owing to the symmetry of the problem, only one quarter of the model is discretized. The simulation results obtained with the SHB elements, in terms of load-displacement curves at the load points A and B, are compared in Fig. 12 with those given by ABAQUS elements as well as with the reference solutions given by Park et al. [30] and Sze et al. [31]. It can be seen once again that the SHB elements perform very well with respect to the reference solutions, which is also the case for ABAQUS prismatic and shell elements. However, as pointed out in the previous nonlinear benchmark problem, a finer mesh is required for the ABAQUS quadratic solid element C3D20, in order to obtain an accurate solution. 


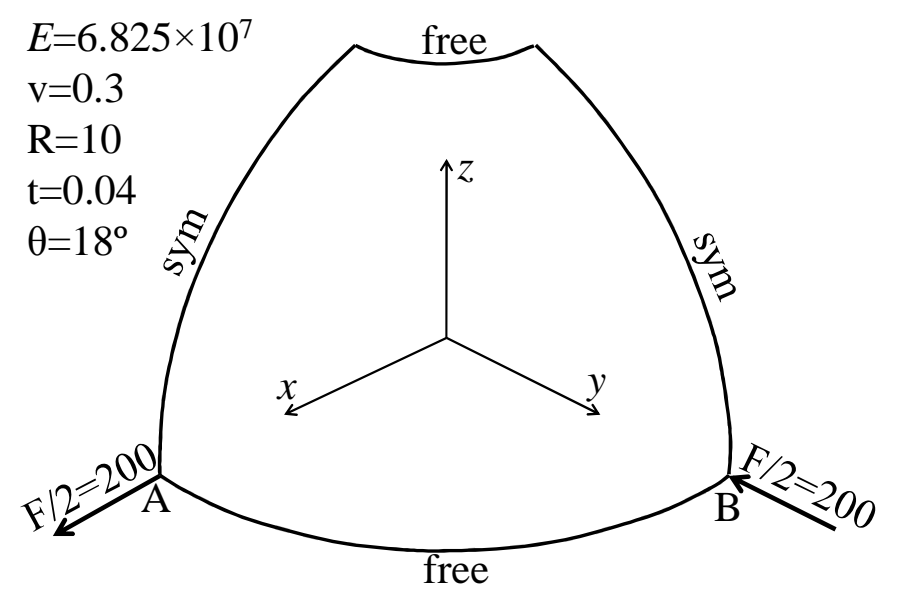

Fig. 11. Geometry, elastic properties, and boundary conditions for the hemispherical shell subjected to alternating radial forces.

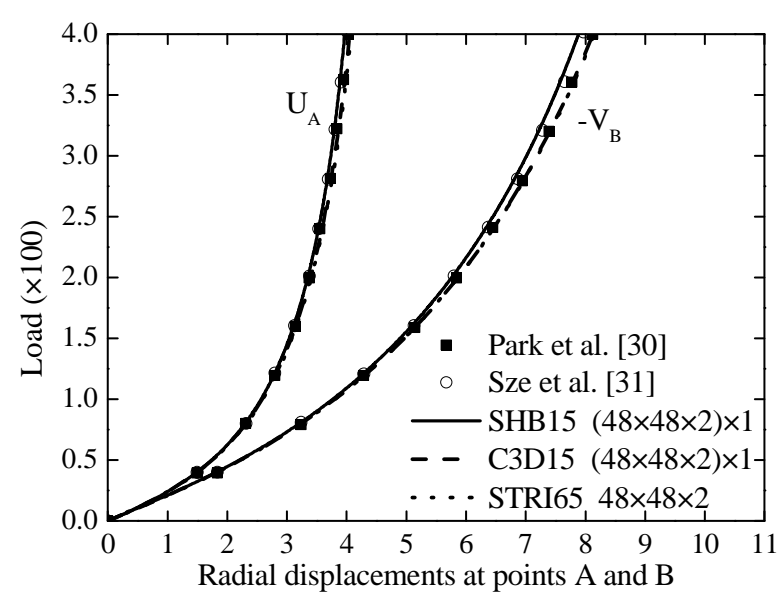

(a) triangular shell / prismatic elements

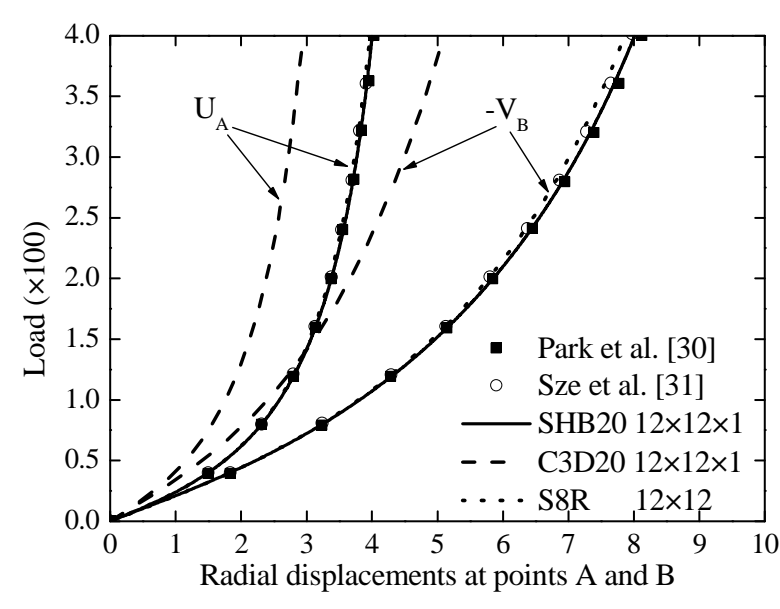

(b) quadrilateral shell / hexahedral elements

Fig. 12. Load-displacement curves at points A and B for the hemispherical shell subjected to alternating radial forces.

\subsection{Cantilever plate subjected to a concentrated force}

An elastic cantilever plate with a concentrated force at one corner, as proposed by Hsiao [32], is considered here. The geometric dimensions, elastic properties, and boundary conditions of the problem are all summarized in Fig. 13. Figure 14 reports the load-displacement curves at the corner point A, in the $x, y$, and $z$ directions (see Fig. 13). In this Fig. 14, the results obtained with the SHB elements are compared with those given by ABAQUS quadratic solid and shell elements, 
on the one hand, and with the reference solutions given by Hsiao [32] and Barut et al. [33], on the other hand. These comparisons reveal that the results given by the SHB elements are in very good agreement with the reference solutions, which is also the case with the ABAQUS quadratic shell elements STRI65 and S8R and the ABAQUS prismatic solid element C3D15. However, adopting the same coarse mesh as that used for the SHB20 and S8R elements (i.e., $4 \times 3 \times 1$ elements), the solution given by the ABAQUS solid element C3D20 falls far from the reference solution, which confirms once again the need for resorting to much finer meshes to achieve an accurate solution.

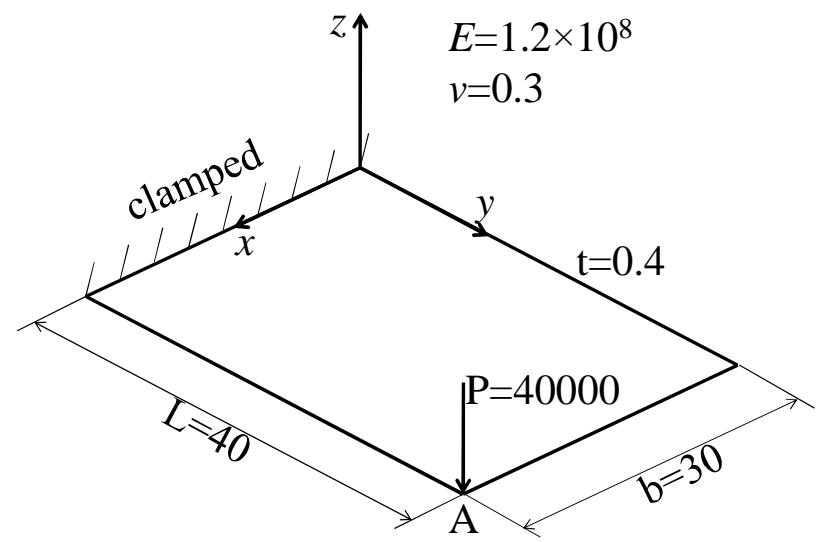

Fig. 13. Geometry, elastic properties, and boundary conditions for the cantilever plate.

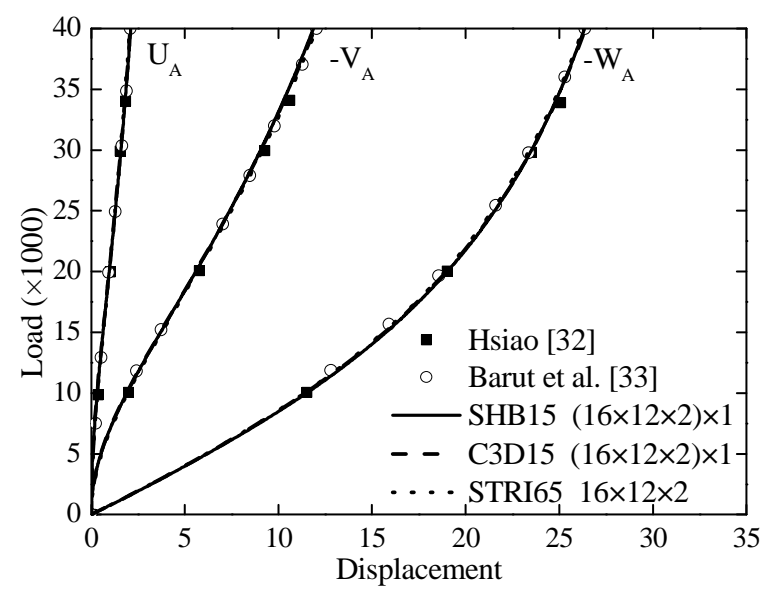

(a) triangular shell / prismatic elements

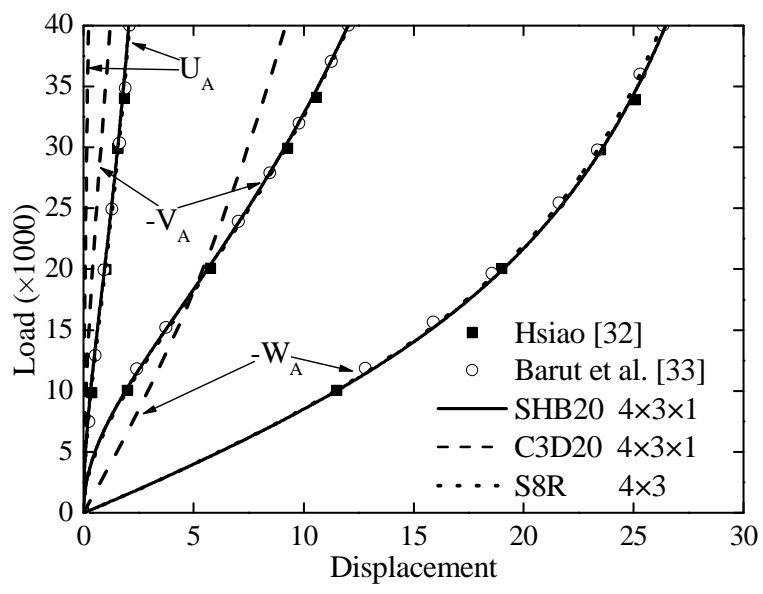

(b) quadrilateral shell / hexahedral elements

Fig. 14. Load-displacement curves for the cantilever plate under a concentrated force. 


\subsection{Bending of a clamped twisted beam}

A clamped twisted beam under out-of-plane loading is analyzed in this section, which is considered as a severe nonlinear benchmark test investigated by a number of authors in the literature (see, e.g., $[12,34,35])$. All geometric dimensions and material properties for this twisted beam problem are specified in Fig. 15. The load-displacement curves at the loading point in the $x, y$, and $z$ directions are reported in Fig. 16. In this Fig. 16, the results obtained with the SHB elements are compared with those given by ABAQUS quadratic solid and shell elements, on the one hand, and with the reference solution given by Mostafa et al. [35], on the other hand. This comparison shows that the results obtained with the SHB elements are in excellent agreement with the reference solution as well as with that given by the ABAQUS quadrilateral shell element S8R. However, taking the same mesh as that used for the SHB15 element, the ABAQUS triangular shell element STRI65 failed to converge in this nonlinear benchmark test, while the C3D15 element provides less accurate results, which reveals the need for a finer mesh. The latter observation is far more critical for the C3D20 element, which provides once again the farthest results with respect to the reference solution.

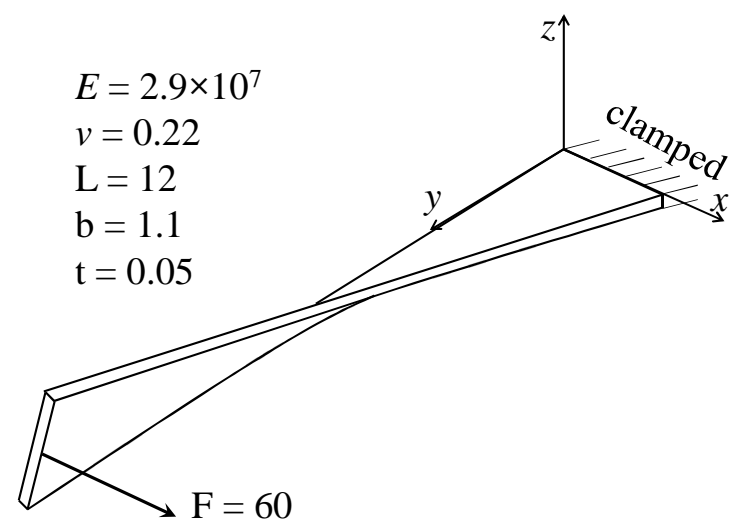

Fig. 15. Geometry, elastic properties, and boundary conditions for the twisted beam subjected to out-of-plane loading. 


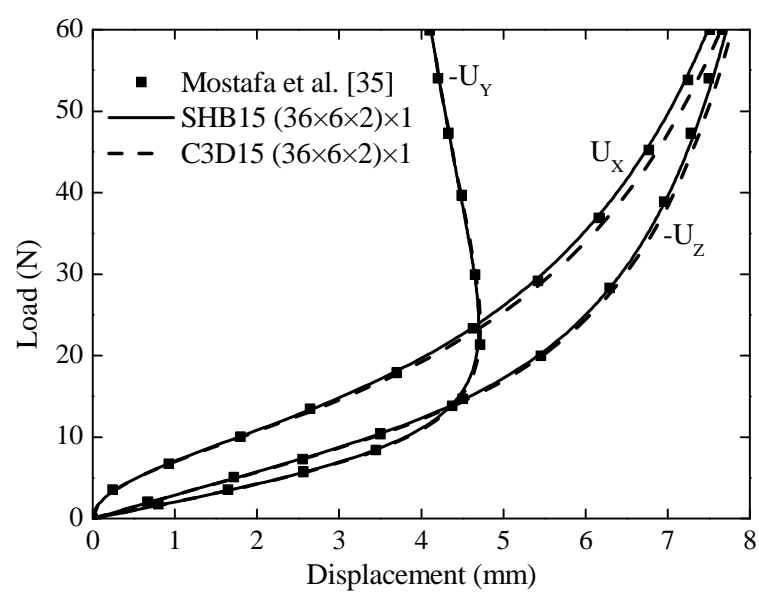

(a) triangular shell / prismatic elements

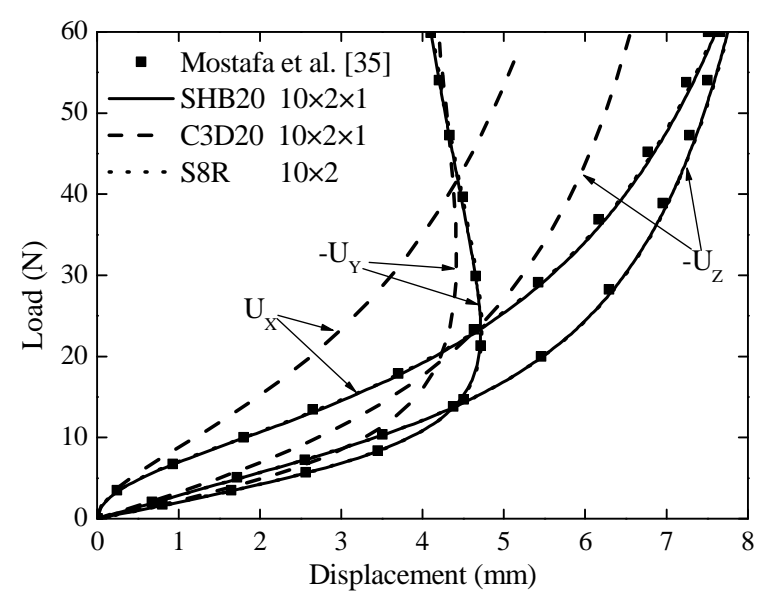

(b) quadrilateral shell / hexahedral elements

Fig. 16. Load-displacement curves for the clamped twisted beam subjected to out-of-plane loading.

\subsection{Bending of a clamped curved beam}

Bending of a curved beam, as illustrated in Fig. 17, is a typical nonlinear benchmark test for beam structures, in which various deformation modes (e.g., tension, bending, shear) are involved (see Smoleński [34]). The three-dimensional displacement at the loading point is investigated using the proposed SHB elements and ABAQUS quadratic elements. The corresponding load-displacement curves are reported in Fig. 18 along with the reference solution given by Smoleński [34]. As can be seen in Fig. 18, the results obtained with the SHB elements are in excellent agreement with the reference solution as well as with those given by ABAQUS quadratic elements, except for the ABAQUS solid element C3D20. For the latter, the results yielded by a coarse mesh (see Fig. 18 (b)) are far from the reference solution, which reveals that the C3D20 quadratic solid element requires much finer meshes to achieve an accurate solution for this nonlinear test problem. 


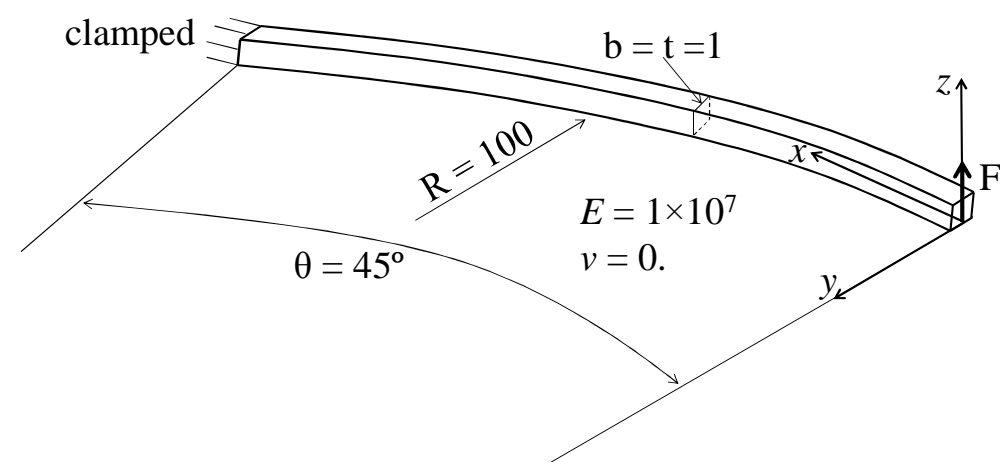

Fig. 17. Geometry, elastic properties, and boundary conditions for the curved beam.

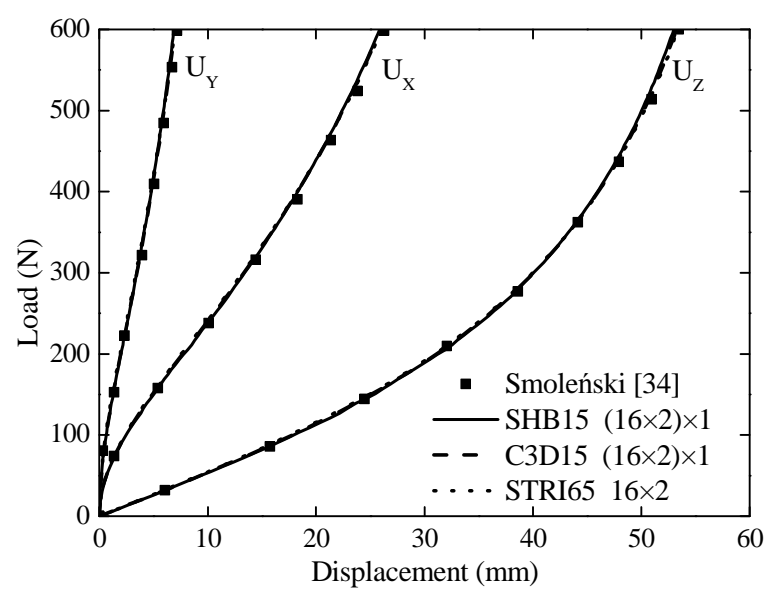

(a) triangular shell / prismatic elements

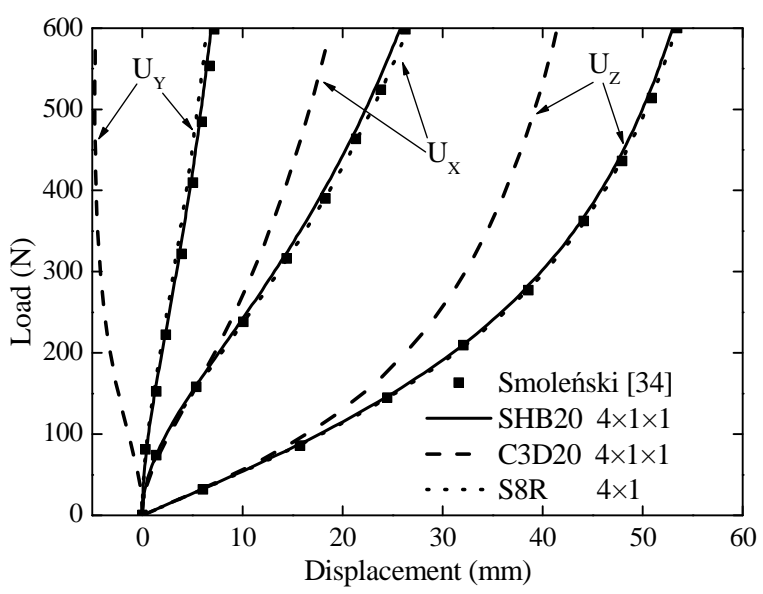

(b) quadrilateral shell / hexahedral elements

Fig. 18. Load-displacement curves for the curved beam under a concentrated force.

\subsection{Inflation of an elastic-perfectly-plastic square plate}

In this test, the inflation of a simply supported square plate, as illustrated in Fig. 19, is considered to evaluate the performance of the proposed SHB elements in the framework of combined geometric and material nonlinearities (i.e., large strains and plastic behavior). The square plate is simply supported at its four edges, and subjected to uniform pressure loading $P=0.6$. The material parameters of the plate corresponding to elastic-perfectly-plastic behavior are summarized in Fig. 19 (see [36-38]). Note that for this nonlinear test, which involves large plastic strains, three integration points through the thickness are required to obtain accurate solutions. Owing to the symmetry of the problem, only one quarter of the square plate is discretized. 


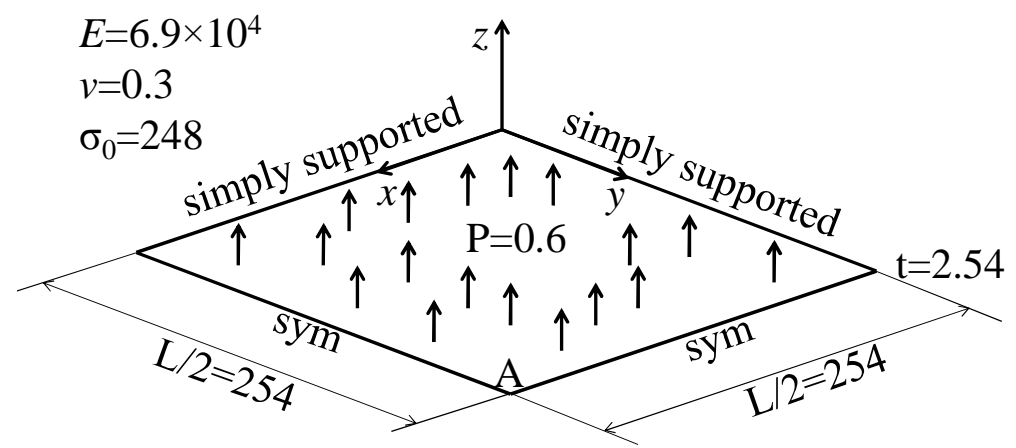

Fig. 19. Geometry, material properties, and boundary conditions for the simply supported square plate subjected to a uniform pressure.

With the increase in the applied pressure, the square plate undergoes a pillow-type deformation mode, as displayed in Fig. 20, where the plastic zones are mainly localized in the four corners. The simulated pressure-displacement curves at the center of the plate are depicted in Fig. 21. It can be seen that the results obtained with the SHB elements are in good agreement with the reference solutions taken from Betsch and Stein [36] and Fontes Valente et al. [37] as well as with those given by ABAQUS quadratic elements, except for the C3D20 solid element and the STRI65 shell element. The latter ABAQUS elements provide results that slightly deviate from the reference solutions (see Fig. 21).

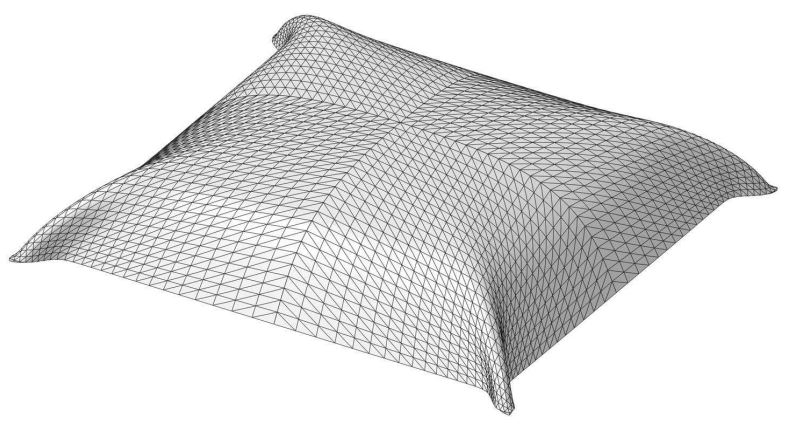

(a) SHB15 elements

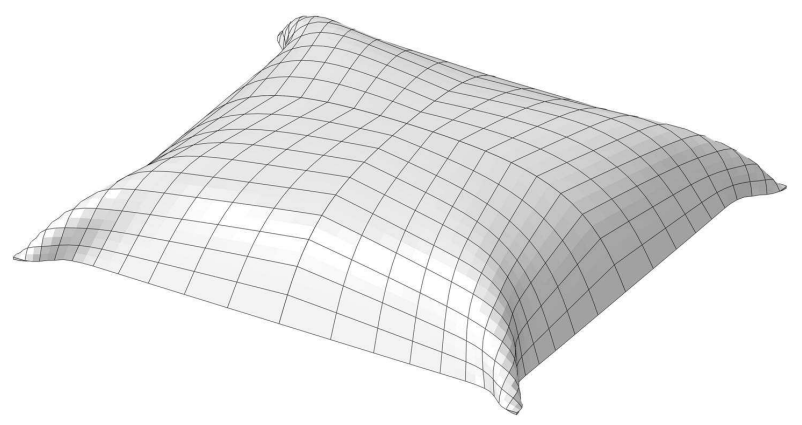

(b) SHB20 elements

Fig. 20. Final deformed shape for the square plate under uniform pressure. 


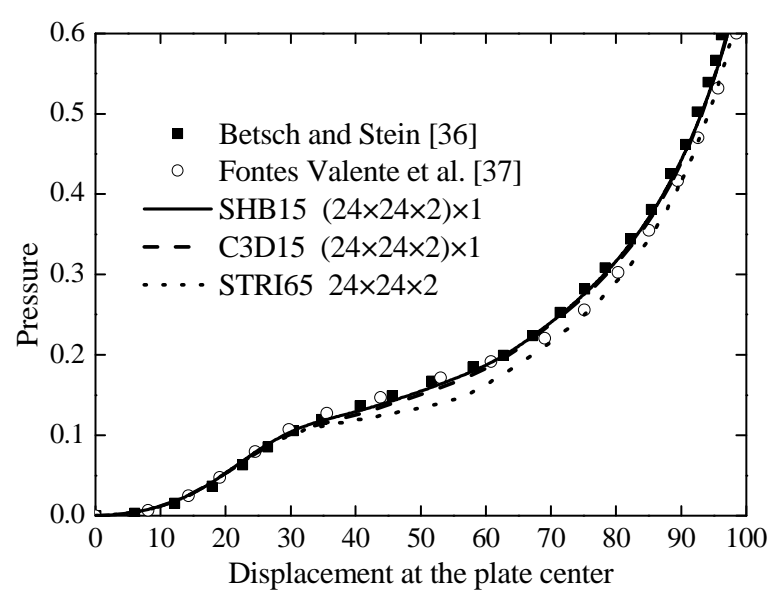

(a) triangular shell / prismatic elements

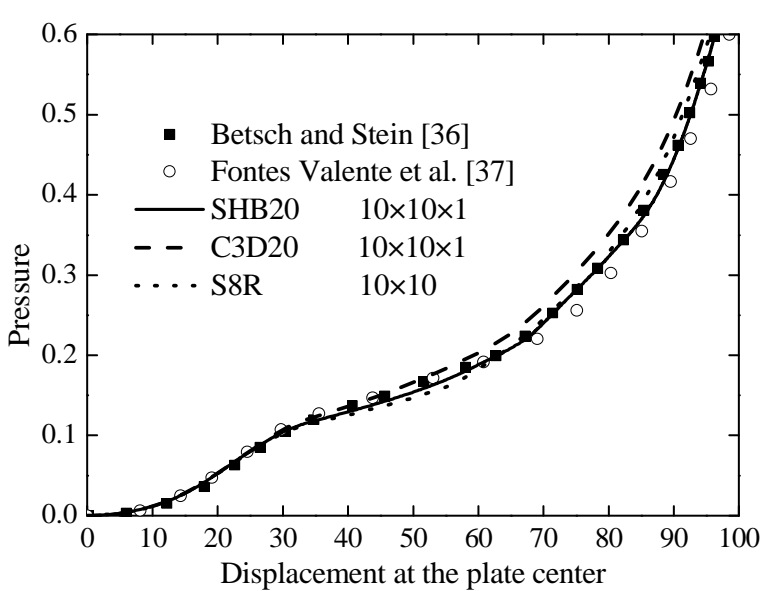

(b) quadrilateral shell / hexahedral elements

Fig. 21. Load-displacement curves at the center point of the plate.

\subsection{Pinched cylinder with rigid end diaphragms}

The second elasto-plastic test consists of a cylinder subjected to two opposite radial forces at its middle and bounded by rigid diaphragms on its ends. This popular benchmark problem has been considered by a number of authors (see, e.g., [37, 39-41]) to assess the performance of finite elements in large plastic strains. The geometric dimensions, material properties, and boundary conditions of the pinched cylinder are all summarized in Fig. 22. In conjunction with the elasto-plastic material behavior, a linear isotropic hardening law is considered. Owing to the symmetry, only one eighth of the cylinder is modeled. 


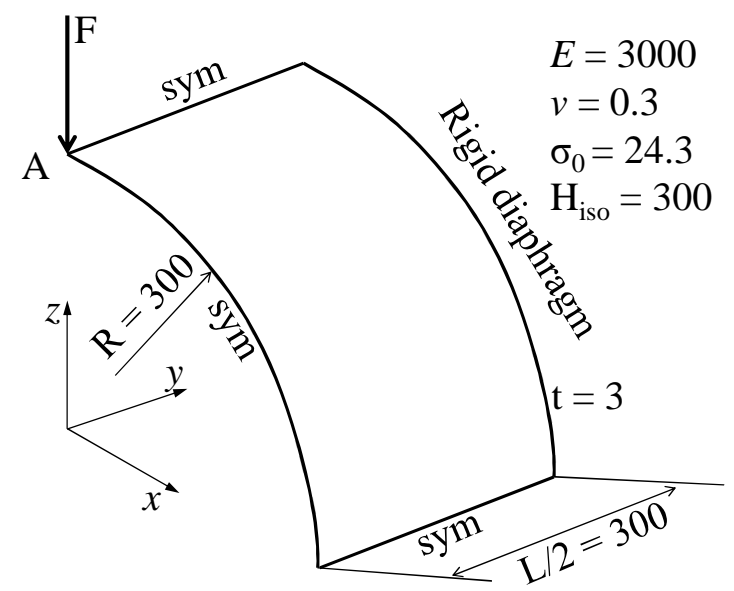

Fig. 22. Geometry, material properties, and boundary conditions for the pinched cylinder.

Figure 23 illustrates the final deformed shape of the pinched cylinder, as obtained with the SHB elements. The simulated force-displacement curves at the loading point A (as denoted in Fig. 22) are reported in Fig. 24 along with the reference solutions taken from Wriggers et al. [39], Eberlein and Wriggers [40] and Hauptmann et al. [41]. It can be seen that the results obtained with the SHB elements are in good agreement with the reference solutions along the entire loading history, which is also the case with the ABAQUS prismatic solid element C3D15 and the ABAQUS shell elements STRI65 and S8R. For the C3D20 quadratic solid element, however, the force-displacement response is well predicted during the elastic stage of loading (up to displacement of $100 \mathrm{~mm}$ ), while the simulated response is overestimated at larger plastic strains (up to $20 \%$ with respect to the reference solutions).

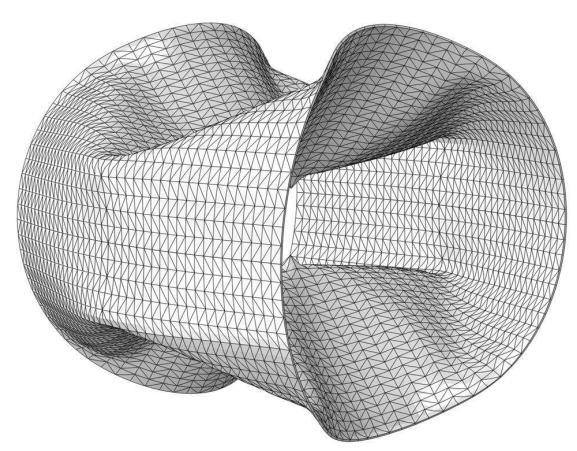

(a) SHB15 elements

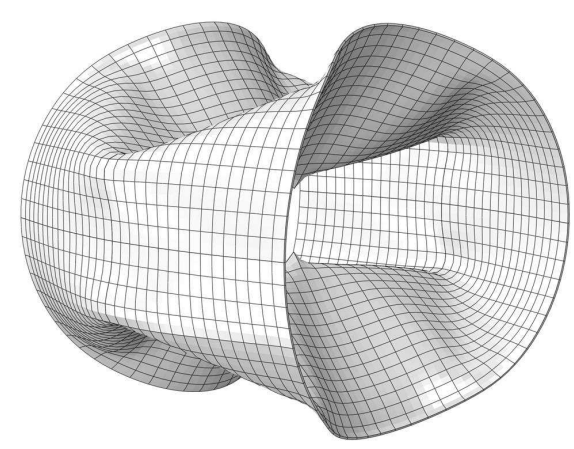

(b) SHB20 elements

Fig. 23. Final deformed shape for the pinched cylinder problem. 


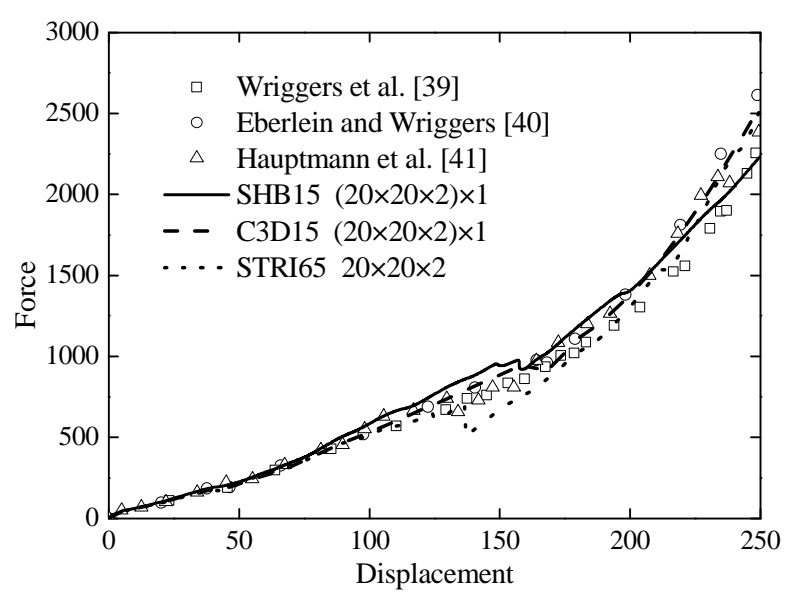

(a) triangular shell / prismatic elements

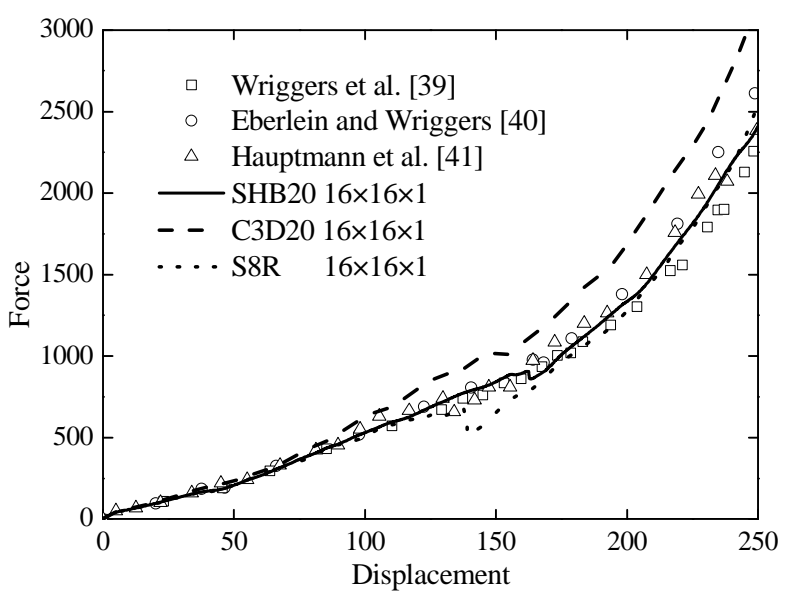

(b) quadrilateral shell / hexahedral elements

Fig. 24. Force-displacement curves at the loading point for the pinched cylinder.

\section{Simulation of sheet metal forming processes}

This section is dedicated to the validation of the proposed SHB elements in the context of sheet metal forming. To this end, a set of selective benchmark problems are simulated with the SHB elements, which consist of three well-known deep drawing tests as well as an incremental forming process. Despite the strong and coupled nonlinearities involved in such applications (i.e., geometric and material nonlinearities as well as contact), only a single element layer, with three through-thickness integration points, is consistently considered throughout this section, for all meshes consisting of SHB elements. The simulation results are compared both with those given by ABAQUS elements and with experiment measurements taken from the literature.

\subsection{Springback simulation of U-shape deep drawing}

The springback simulation of the U-shape deep drawing process has been proposed as a benchmark test by the sheet metal forming community in the NUMISHEET'93 conference [42]. The schematic view of the setup and its geometric dimensions are described in Fig. 25. All details regarding the simulation process can be found in the related literature (see, e.g., [16, 43-45]). This deep drawing process is divided into two steps: the forming step, followed by the springback step. During the first step, the U-shape is formed until the maximum punch stroke of $70 \mathrm{~mm}$ is 
reached under a holding force of $2.45 \mathrm{kN}$ (see Fig. 26 (a)). Then, the springback stage of the sheet takes place by removing the holding force and all contact between the sheet and the tools (see Fig. 26 (b)).

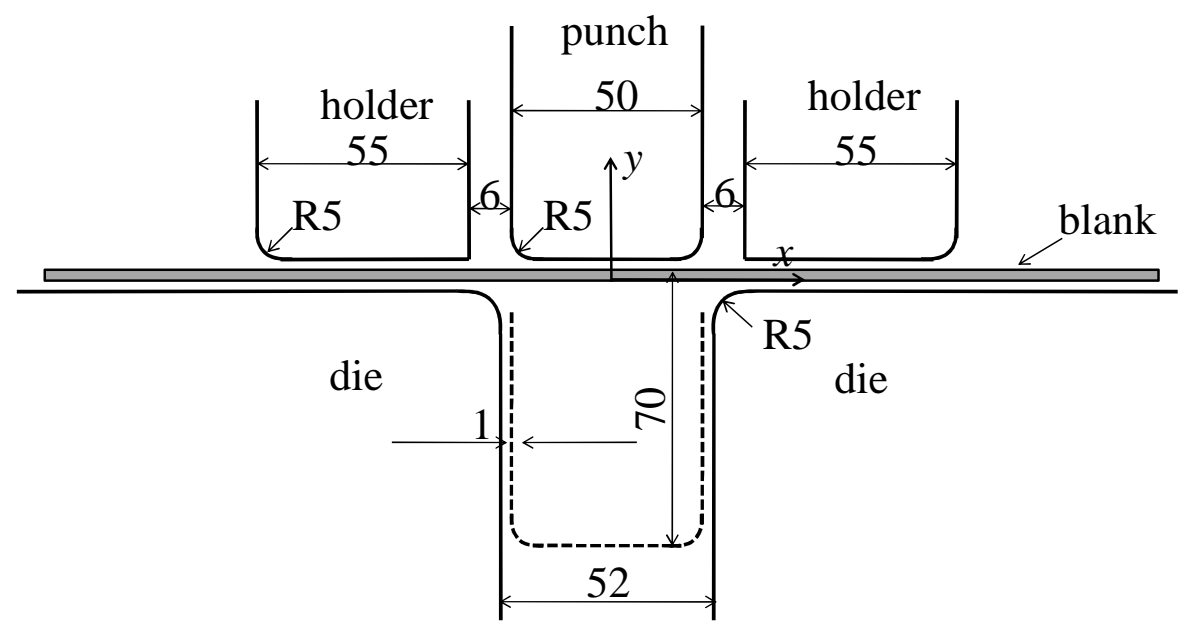

Fig. 25. Setup of the U-bending tools.

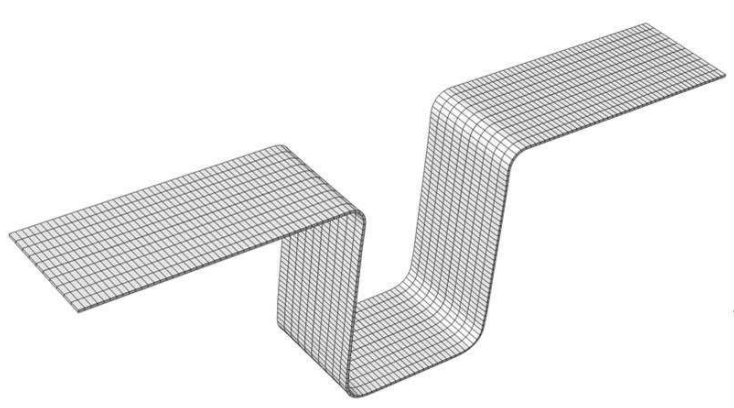

(a) end of forming step

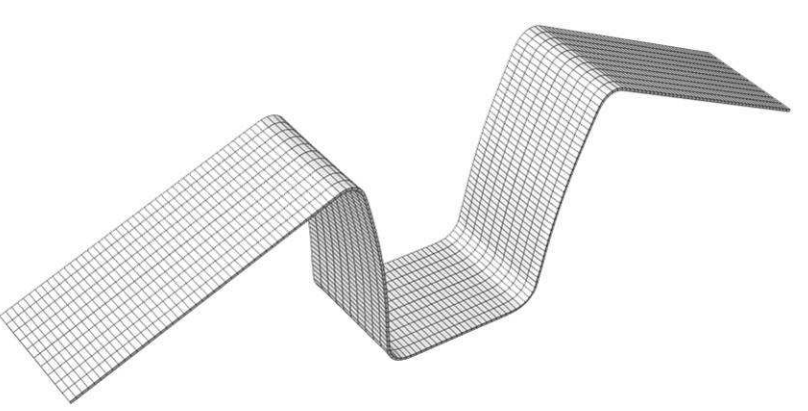

(b) after springback

Fig. 26. Illustration of the deformed sheet, in the U-shape deep drawing test, at (a) the end of forming step, and (b) after springback.

Both an aluminum-alloy sheet and a steel sheet are considered in this study. The initial dimensions of the aluminum sheet are $350 \mathrm{~mm} \times 35 \mathrm{~mm} \times 0.81 \mathrm{~mm}$, with a friction coefficient between the tools and the blank equal to 0.162, while the initial dimensions of the steel sheet are $350 \mathrm{~mm} \times 35 \mathrm{~mm} \times 0.78 \mathrm{~mm}$, with a friction coefficient equal to 0.144 . The elasto-plastic 
parameters associated with both materials are summarized in Table 4, in which the following Swift law has been considered to describe isotropic hardening

$Y=k\left(\varepsilon_{0}+\varepsilon_{e q}^{p}\right)^{n}$

where $\varepsilon_{e q}^{p}$ is the equivalent plastic strain (see the plastic yield function defined in Eq. (20)).

Table 4

Elastic properties and Swift's isotropic hardening parameters.

\begin{tabular}{lccccc}
\hline Material & $E(\mathrm{MPa})$ & $v$ & $\varepsilon_{0}$ & $k(\mathrm{MPa})$ & $n$ \\
\hline Aluminum & 71,000 & 0.33 & 0.01658 & 576.79 & 0.3593 \\
Steel & 206,000 & 0.3 & 0.007117 & 565.32 & 0.2589 \\
\hline
\end{tabular}

The anisotropic plastic behavior of the materials is taken into account by considering the Hill [25] quadratic yield criterion. The Lankford coefficients associated with both studied materials are listed in Table 5.

\section{Table 5}

Lankford's coefficients for both studied materials.

\begin{tabular}{lccc}
\hline Material & $r_{0}$ & $r_{45}$ & $r_{90}$ \\
\hline Aluminum & 0.71 & 0.58 & 0.70 \\
\hline Steel & 1.79 & 1.51 & 2.27 \\
\hline
\end{tabular}

Considering the symmetry of the problem, only one quarter of the blank is analyzed. The latter is discretized by $(100 \times 5 \times 2) \times 1$ triangular shell or prismatic elements and $100 \times 5 \times 1$ hexahedral elements, respectively (the mesh nomenclature is the same as that used in Section 3). As stated before, only three integration points through the thickness are considered in the simulations using the SHB and ABAQUS elements. Note that the simulations with the ABAQUS quadratic shell element S8R failed to converge for both studied materials, which clearly emphasizes the 
limitations of this shell element in handling double-sided contact in sheet metal forming processes.

To quantify the amount of springback for the blank after the forming stage, the angles around the punch radius and the die radius ( $\theta_{1}$ and $\theta_{2}$, respectively, in Fig. 27) are investigated. The simulation results obtained with the SHB elements are compared in Tables 6 and 7 with those given by ABAQUS elements as well as with experimental measurements and numerical solutions available in the literature. On the whole, the angles after springback predicted with the SHB elements are in good agreement with those given by ABAQUS elements, and lie in the intervals defined by the reference results. These results demonstrate the good capabilities of the SHB elements in modeling sheet metal forming processes, where various nonlinearities (geometric, material, and double-sided contact) enter into play, while using only a single element layer with few through-thickness integration points.

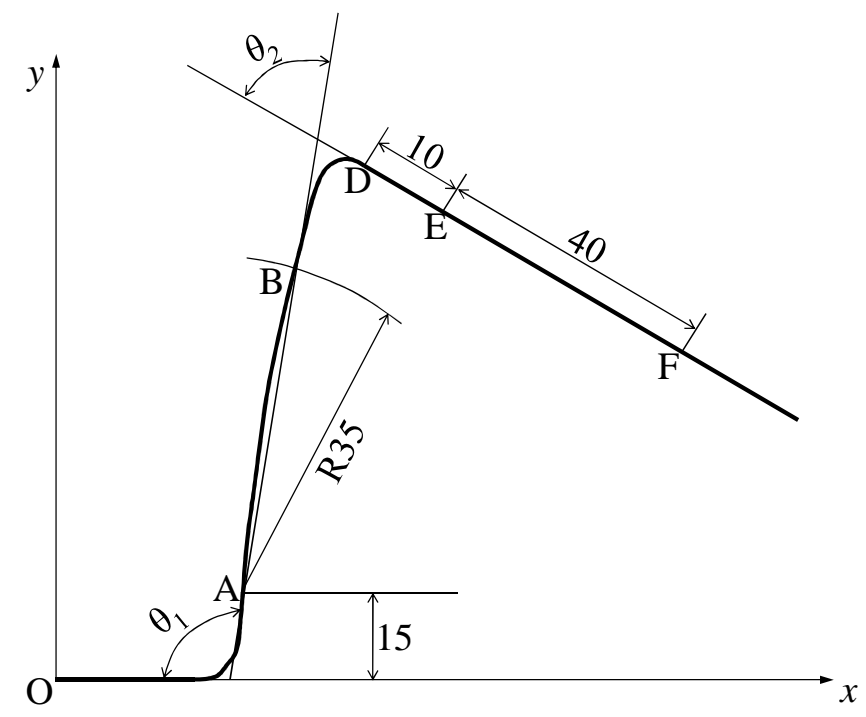

Fig. 27. Definition of springback angles $\theta_{1}$ and $\theta_{2}$. 
Table 6

Springback angles $\theta_{1}$ and $\theta_{2}$ for the aluminum material.

\begin{tabular}{ccccccccc}
\hline Material & Angle $\left({ }^{\circ}\right)$ & Experiment* $^{*}$ & Simulation* & STRI65 & C3D15 & SHB15 & C3D20 & SHB20 \\
\hline \multirow{2}{*}{ Aluminum } & $\theta_{1}$ & $101.5^{\circ} \sim 116.0^{\circ}$ & $62.0^{\circ} \sim 134.0^{\circ}$ & $107.28^{\circ}$ & $108.87^{\circ}$ & $102.72^{\circ}$ & $106.03^{\circ}$ & $104.13^{\circ}$ \\
& $\theta_{2}$ & $68.5^{\circ} \sim 77.5^{\circ}$ & $63.0^{\circ} \sim 91.0^{\circ}$ & $69.85^{\circ}$ & $69.67^{\circ}$ & $70.74^{\circ}$ & $70.74^{\circ}$ & $74.53^{\circ}$ \\
\hline
\end{tabular}

* Note: The experimental and simulated intervals are given in Flores [16].

Table 7

Springback angles $\theta_{1}$ and $\theta_{2}$ for the steel material.

\begin{tabular}{ccccccccc}
\hline Material & Angle $\left(^{\circ}\right)$ & Reference $^{*}$ & Reference $3^{*}$ & STRI65 & C3D15 & SHB15 & C3D20 & SHB20 \\
\hline \multirow{2}{*}{ Steel } & $\theta_{1}$ & $101.06^{\circ}$ & $100.82^{\circ}$ & $97.94^{\circ}$ & $99.67^{\circ}$ & $99.37^{\circ}$ & $97.03^{\circ}$ & $98.32^{\circ}$ \\
& $\theta_{2}$ & $79.99^{\circ}$ & $80.45^{\circ}$ & $80.10^{\circ}$ & $80.27^{\circ}$ & $81.05^{\circ}$ & $82.33^{\circ}$ & $82.52^{\circ}$
\end{tabular}

* Note: Reference 2 corresponds to Dvorkin and Bathe [45], while reference 3 refers to Park and Oh [43].

\subsection{Single point incremental sheet metal forming}

For the past two decades, the incremental forming technology has attracted much attention due to its advantages in terms of economical operability. Single Point Incremental Forming (SPIF) has become a typical test in the context of incremental forming process (see, e.g., Bouffioux et al., [46], Sena et al. [47]). As illustrated in Fig. 28, a clamped square sheet is gradually deformed in its central area by applying a spherical punch with a radius of $5 \mathrm{~mm}$ following a preset path. The punch is initially set to be tangent to the sheet surface, and located $41 \mathrm{~mm}$ away from one side of the sheet. The whole forming process consists in the following five steps: 1) the punch indents the sheet with $5 \mathrm{~mm}$ depth along the $y$-direction; 2) the punch moves at the same depth following a line of $100 \mathrm{~mm}$ along the $x$-direction; 3 ) the punch indents a second time the sheet up to a depth of $10 \mathrm{~mm}$; 4) the punch moves back, at the same new depth, following a line of $100 \mathrm{~mm}$ along 
the $x$-direction; 5) and finally an unloading step takes place, with the punch returning back to its initial position.

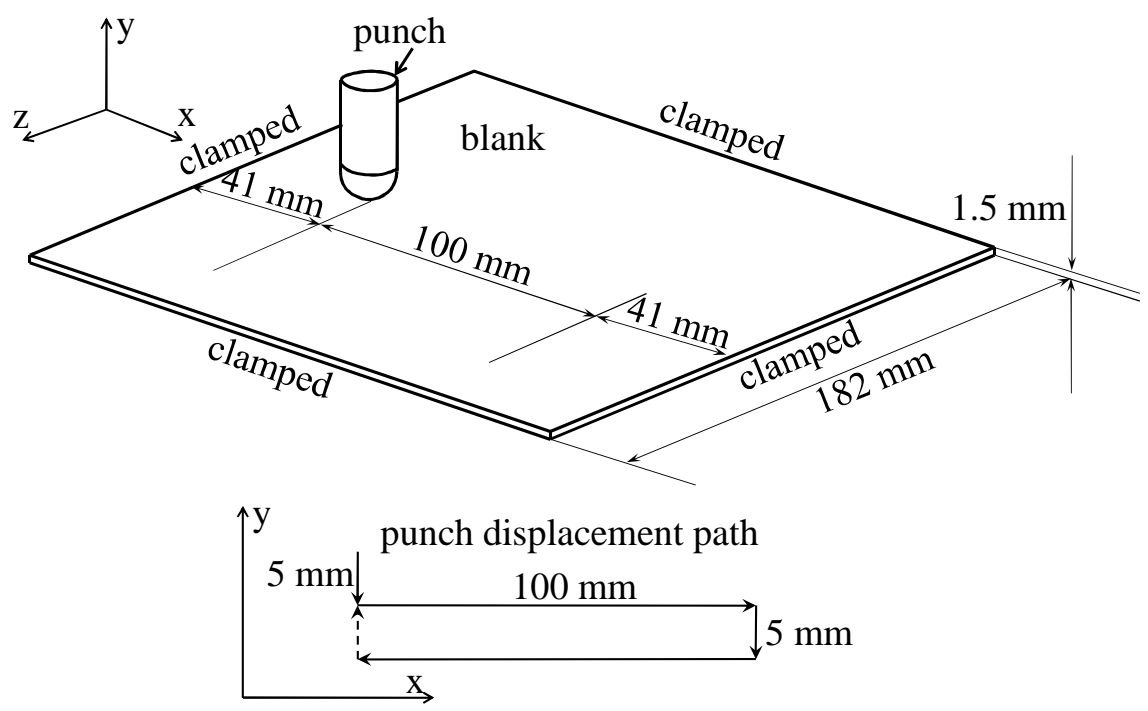

Fig. 28. Description of the single point incremental forming test.

The material used for the simulations is an aluminum alloy AA3103-O (see Bouffioux et al. [46]). The associated elasto-plastic material parameters are summarized in Table 8, according to the Swift isotropic hardening law (see Eq. (26)).

\section{Table 8}

Material parameters for the AA3103-O aluminum alloy.

\begin{tabular}{lccccc}
\hline Material & $E(\mathrm{MPa})$ & $v$ & $\varepsilon_{0}$ & $k(\mathrm{MPa})$ & $n$ \\
\hline AA3103-O & 72,600 & 0.36 & 0.00057 & 180 & 0.229 \\
\hline
\end{tabular}

The contact conditions between the punch and the sheet are assumed frictionless. Because the sheet is deformed mainly in the central area, only one half of the model is meshed with $(60 \times 15 \times 2) \times 1$ quadratic elements, in the case of prismatic elements, and $60 \times 15 \times 1$ quadratic elements, in the case of hexahedral elements (again, the mesh nomenclature is the same as that used in Section 3). The obtained results in terms of punch force-punch displacement correspond to converged solutions using only a single element layer with three through-thickness integration 
points. Figure 29 shows the final deformed mesh for this SPIF test, as obtained with the proposed SHB elements.

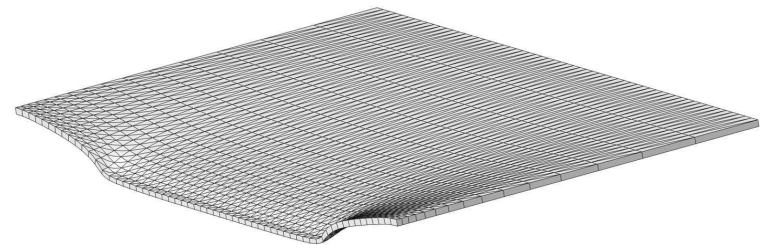

(a) $(60 \times 15 \times 2) \times 1$ SHB 15 elements

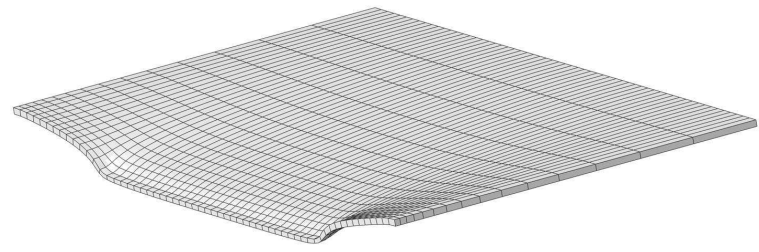

(b) $60 \times 15 \times 1$ SHB20 elements

Fig. 29. Final deformed shape for the SPIF sheet.

The punch force-punch displacement responses simulated with the proposed SHB elements are compared in Fig. 30 with those given by ABAQUS elements as well as with the experimental results provided by Bouffioux et al. [46]. It should be noted that the simulations using ABAQUS shell elements (i.e., STRI65 and S8R) failed to converge, due to contact-type nonlinearities. This suggests, once again, that finite element formulations based on fully 3D approaches are more appropriate to model sheet metal forming processes in which double-sided contact enters into play. As shown in Fig. 30, the punch force-punch displacement responses obtained with the SHB elements are in good agreement with those given by ABAQUS elements, although both lying higher than the experimental results. Note that oscillations in the simulated curves are observed both for the SHB elements and for ABAQUS elements. Indeed, when the punch slides during the forming process, the contact between the punch and the sheet within a confined zone is lost and recovered several times, until another new zone comes into the same situation. This phenomenon causes oscillations in the punch force-punch displacement curve, whose amplitudes and number depend on the mesh size of the punch and the sheet (see, e.g., [48]). In order to reduce these numerical oscillations, a refined mesh for the sheet is used in the simulations, which consists of $(100 \times 30 \times 2) \times 1$ quadratic elements, in the case of prismatic elements, and $100 \times 30 \times 1$ quadratic elements, in the case of hexahedral elements. Figure 31 reports the results obtained by using refined meshes, where it is clearly shown that the simulated punch force-punch displacement curves are much smoother than those obtained with coarse meshes. 


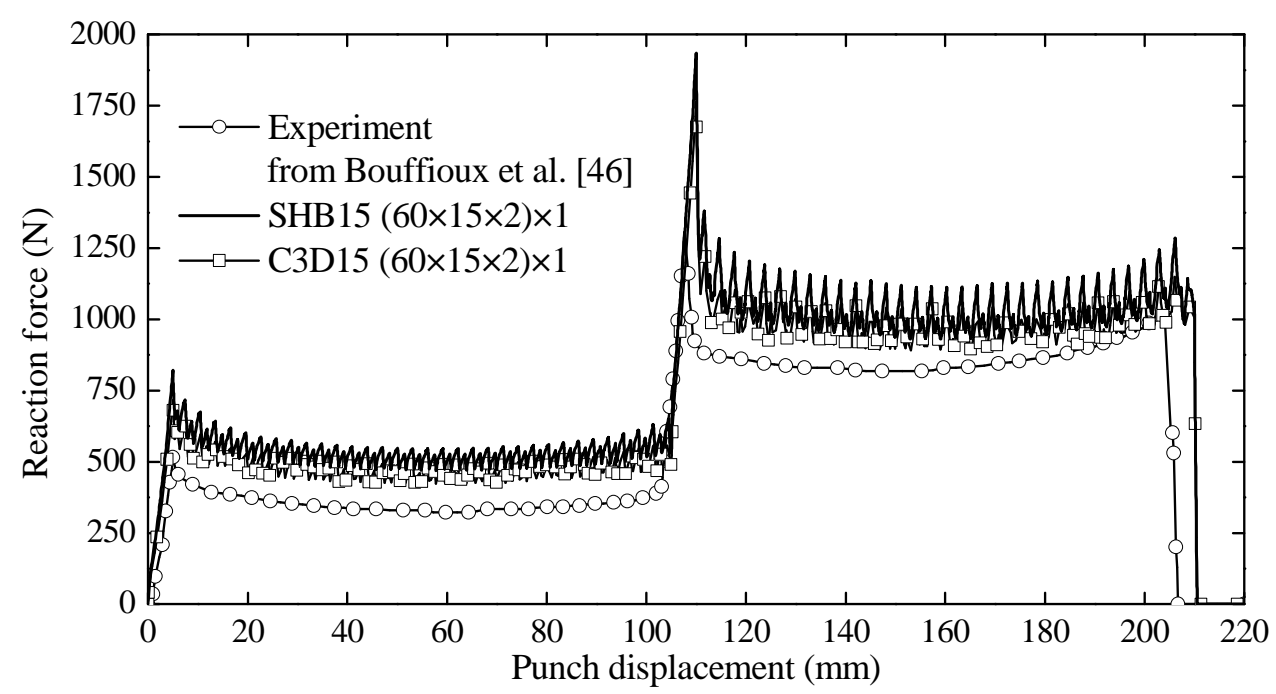

(a) prismatic elements

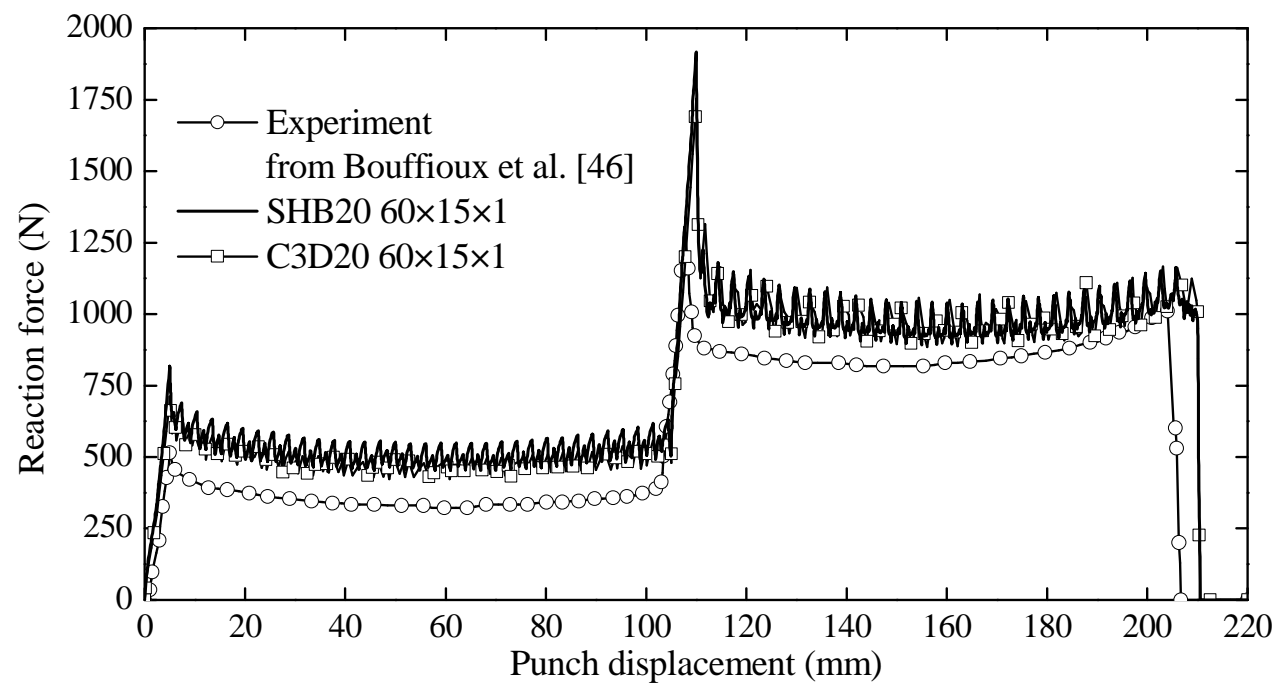

(b) hexahedral elements

Fig. 30. Simulation results using coarse meshes, in terms of punch force evolution for the SPIF test, along with experiments taken from Bouffioux et al. [46]. 


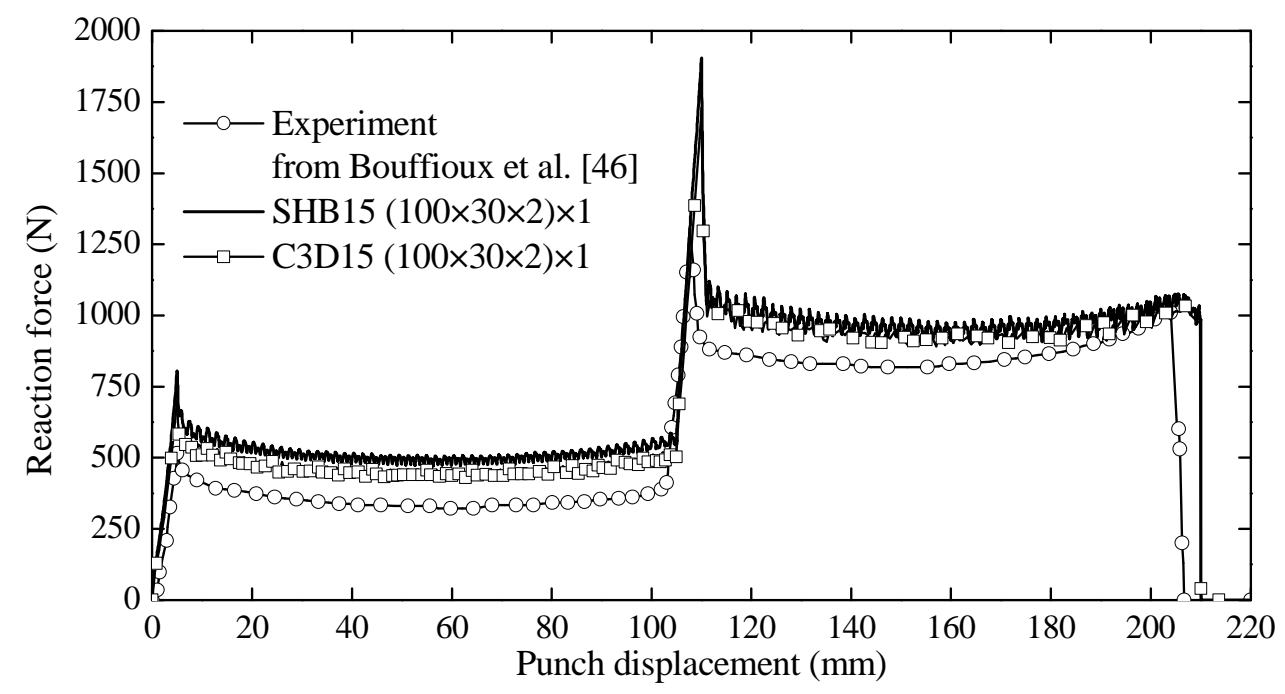

(a) prismatic elements

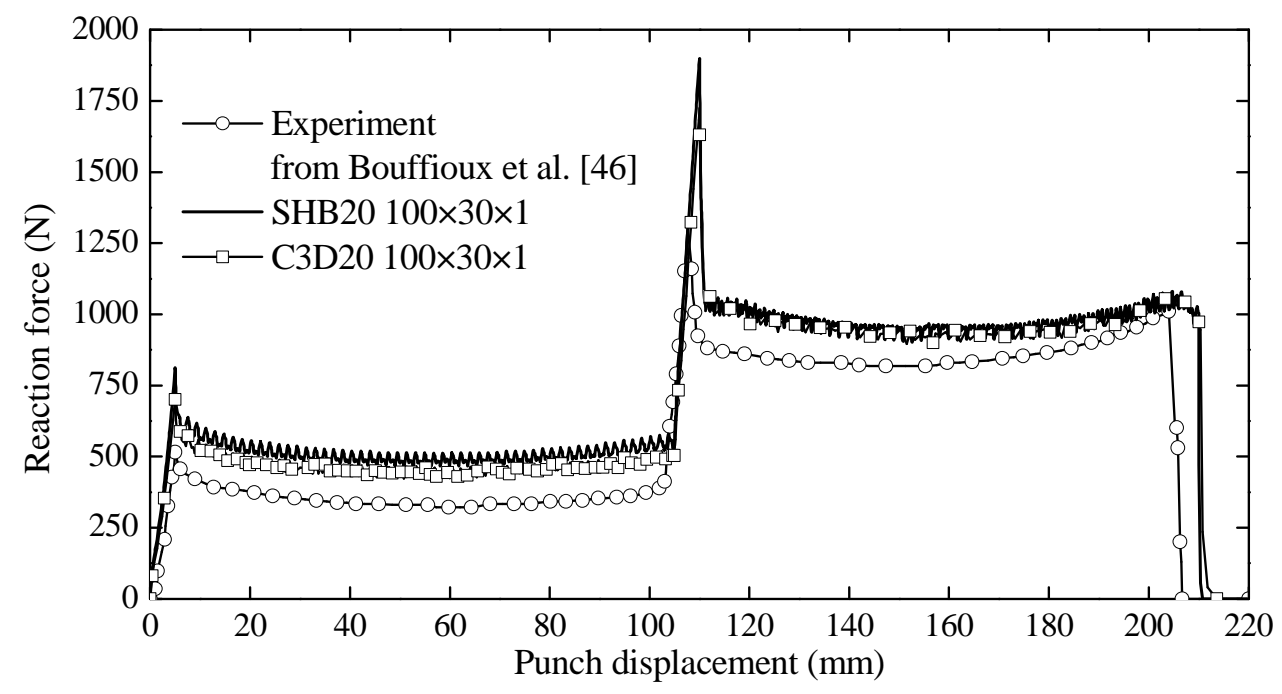

(b) hexahedral elements

Fig. 31. Simulation results using fine meshes, in terms of punch force evolution for the SPIF test, along with experiments taken from Bouffioux et al. [46].

\subsection{Deep drawing of a square cup}

The benchmark test of deep drawing of a square cup, as proposed in the Numisheet'93 conference [42] and subsequently by several other authors (see, e.g., Schwarze et al. [18], Xu et al. [49]), is considered here to assess the ability of the proposed quadratic solid-shell elements to model challenging industrial forming applications. The geometric dimensions of the forming tools are specified in Fig. 32. Two square sheets, with initial dimensions of $150 \times 150 \times 0.81 \mathrm{~mm}$ 
and $150 \times 150 \times 0.78 \mathrm{~mm}$, corresponding to aluminum sheet and steel sheet, respectively, are considered for the simulations. The associated elasto-plastic material parameters, according to the Swift isotropic hardening law (see Eq. 26) and the von Mises yield surface, are the same as those reported in Table 4.

All along the forming process, a constant blank holding force of $16.6 \mathrm{kN}$ is applied. The friction coefficient between the blank and the forming tools is taken equal to 0.162 for the aluminum sheet, and 0.144 for the steel sheet. Owing to the symmetry, only one quarter of the sheets is discretized with a mesh of $(32 \times 32 \times 2) \times 1$ quadratic elements, in the case of prismatic elements, and $32 \times 32 \times 1$ quadratic elements, in the case of hexahedral elements (note that the mesh nomenclature is the same as that used in Section 3). Also, only a single element layer is used in the simulations with three integration points through the thickness. The final deformed shapes of the sheets, which correspond to a maximum punch stroke of $15 \mathrm{~mm}$ for the aluminum square cup and $40 \mathrm{~mm}$ for the steel square cup, are shown in Figs. 33 and 34, respectively.

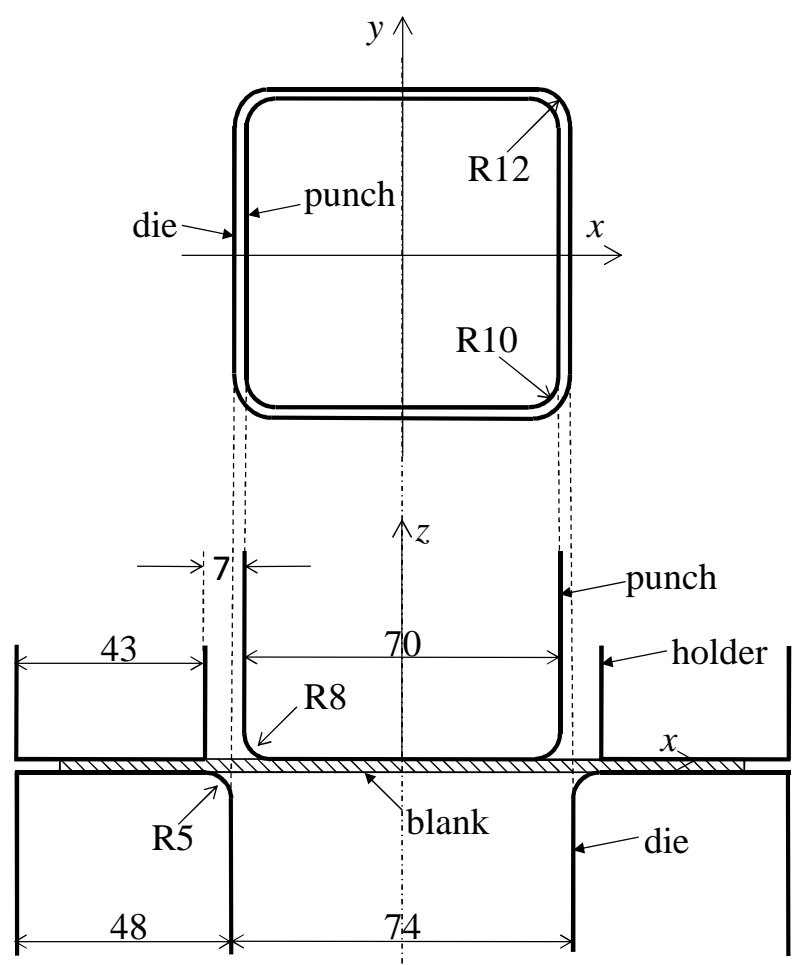

Fig. 32. Schematic view for the square cup drawing process. 


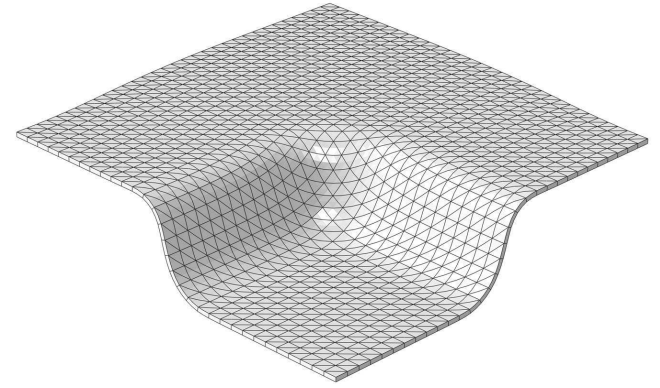

(a) $(32 \times 32 \times 2) \times 1$ SHB 15 elements

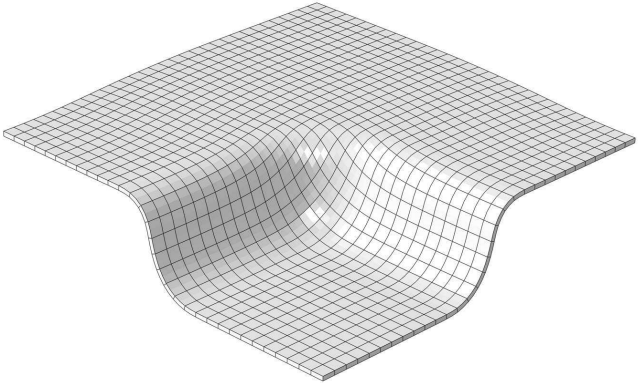

(b) $32 \times 32 \times 1$ SHB20 elements

Fig. 33. Final deformed shape for the aluminum cup at $15 \mathrm{~mm}$ punch stroke.

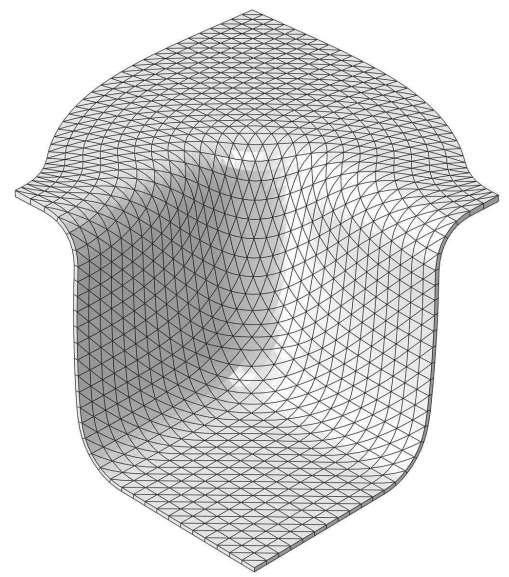

(a) $(32 \times 32 \times 2) \times 1$ SHB 15 elements

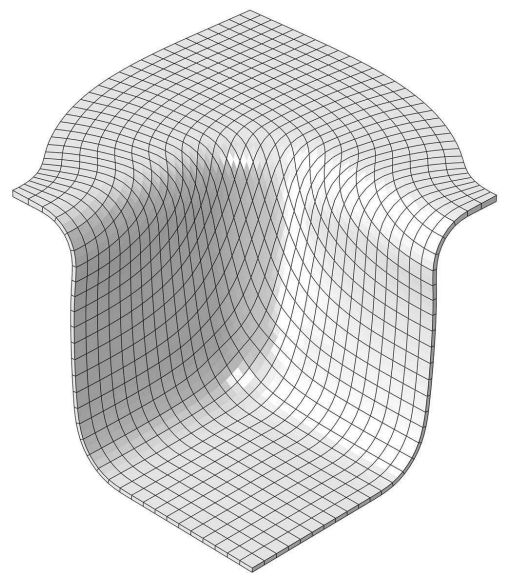

(b) $32 \times 32 \times 1$ SHB20 elements

Fig. 34. Final deformed shape for the steel cup at $40 \mathrm{~mm}$ punch stroke.

Three draw-in distances $D_{x}, D_{y}$, and $D_{d}$, corresponding to the final formed cups, as illustrated in Fig. 35, are investigated here for both studied materials. The predicted results for the aluminum cup and the steel cup are reported in Tables 9 and 10, respectively. Note that, for the same reasons discussed in the previous sheet metal forming processes, the simulations using the ABAQUS quadratic shell elements STRI65 and S8R failed to converge and, accordingly, no results are reported for these elements.

For the aluminum cup, all draw-in distances predicted with the SHB elements lie in the range delimited by the maximum and minimum experimental measurements, which is also the case for the predictions using ABAQUS quadratic solid elements. Note also that the predicted draw-in 
distances $D_{x}$ and $D_{y}$ are identical for all simulations, which is consistent with the assumed isotropic plastic behavior of the sheets.

With respect to the experimental range for the steel cup, the predicted draw-in distances $D_{x}$ and $D_{y}$ are very slightly overestimated with the SHB elements, while they are very slightly underestimated with the ABAQUS solid elements. Nevertheless, the diagonal draw-in distance $D_{d}$ predicted with the SHB elements is in good agreement with the experimental measurements, whereas it is somewhat underestimated with the ABAQUS solid elements. On the whole, the proposed quadratic solid-shell elements perform better than their ABAQUS counterparts, considering the above-discussed results and the fact that the latter require more integration points (IPs) per element (e.g., 27 IPs for the C3D20 element, compared to only 12 IPs for the SHB20 element).

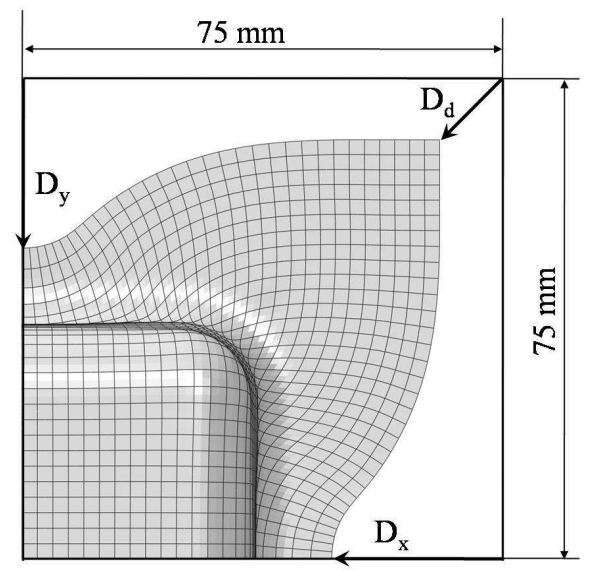

Fig. 35. Definition of the draw-in distances for the final formed square cup. 
Table 9

Draw-in distances for the aluminum cup at $15 \mathrm{~mm}$ punch stroke.

\begin{tabular}{|c|c|c|c|c|c|}
\hline & Mesh & $\begin{array}{c}\text { Number of IPs } \\
\text { per element }\end{array}$ & $\mathrm{D}_{\mathrm{x}}[\mathrm{mm}]$ & $\mathrm{D}_{\mathrm{y}}[\mathrm{mm}]$ & $\mathrm{D}_{\mathrm{d}}[\mathrm{mm}]$ \\
\hline Min. experiment & 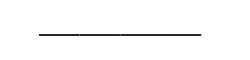 & 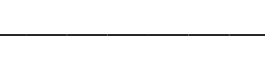 & 3.80 & 3.90 & 2.30 \\
\hline Max. experiment & thes & - & 6.45 & 6.49 & 3.79 \\
\hline C3D15 & $(32 \times 32 \times 2) \times 1$ & 9 & 5.52 & 5.52 & 2.59 \\
\hline SHB15 & $(32 \times 32 \times 2) \times 1$ & 9 & 5.87 & 5.87 & 3.00 \\
\hline $\mathrm{C} 3 \mathrm{D} 20$ & $32 \times 32 \times 1$ & 27 & 5.31 & 5.31 & 2.39 \\
\hline SHB20 & $32 \times 32 \times 1$ & 12 & 5.61 & 5.61 & 2.32 \\
\hline
\end{tabular}

Table 10

Draw-in distances for the steel cup at $40 \mathrm{~mm}$ punch stroke.

\begin{tabular}{ccccccc}
\hline & Mesh & $\begin{array}{c}\text { Number of IPs } \\
\text { per element }\end{array}$ & $\mathrm{D}_{\mathrm{x}}[\mathrm{mm}]$ & $\mathrm{D}_{\mathrm{y}}[\mathrm{mm}]$ & $\mathrm{D}_{\mathrm{d}}[\mathrm{mm}]$ \\
\cline { 3 - 6 } Min. experiment & - & & 26.75 & 26.75 & 14.06 \\
Max. experiment & & & 29.60 & 29.58 & 16.31 \\
C3D15 & $(32 \times 32 \times 2) \times 1$ & 9 & 26.57 & 26.57 & 13.64 \\
SHB15 & $(32 \times 32 \times 2) \times 1$ & 9 & 29.64 & 29.64 & 15.89 \\
C3D20 & $32 \times 32 \times 1$ & 27 & 26.57 & 26.57 & 13.73 \\
SHB20 & $32 \times 32 \times 1$ & 12 & 29.81 & 29.81 & 15.63 \\
\hline
\end{tabular}

\subsection{Deep drawing of a cylindrical cup}

The deep drawing of a cylindrical cup is considered as one of the most popular benchmark problems in the context of sheet metal forming processes. In particular, this test has been often adopted for the analysis of the earing evolution of the cup when anisotropic plastic behavior of 
metal sheets is considered (see, e.g., [50-53]). The initially circular sheet is made of an AA2090T3 aluminum alloy with an initial thickness of $1.6 \mathrm{~mm}$. The plastic behavior of the sheet is described by the Swift isotropic hardening law (see Eq. (26)) together with the Hill [25] anisotropic yield surface. The associated material parameters are summarized in Table 11. The schematic view of the drawing setup and the dimensions of the forming tools are shown in Fig. 36.

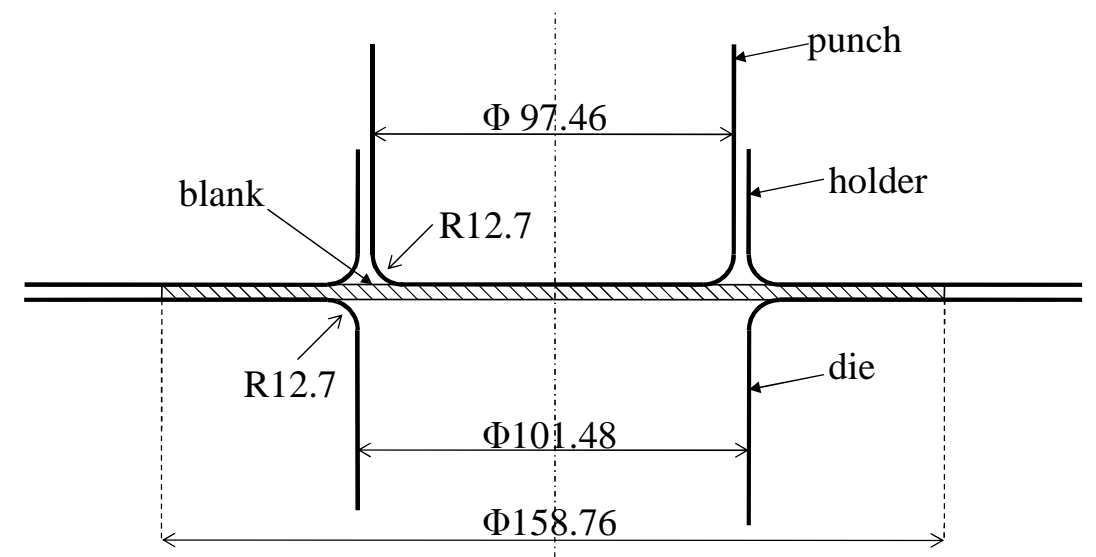

Fig. 36. Schematic view for the cylindrical cup drawing process.

Table 11

Material parameters for the AA2090-T3 aluminum alloy.

\begin{tabular}{lcccccccc}
\hline Material & $E(\mathrm{MPa})$ & $v$ & $\varepsilon_{0}$ & $k(\mathrm{MPa})$ & $n$ & $r_{0}$ & $r_{45}$ & $r_{90}$ \\
\hline AA2090-T3 & 70,500 & 0.34 & 0.025 & 646 & 0.227 & 0.2115 & 1.5769 & 0.6923 \\
\hline
\end{tabular}

Considering the symmetry of the problem, only one quarter of the model is analyzed. Similar to the previously investigated sheet metal forming processes, only the results obtained with the SHB elements and ABAQUS quadratic solid elements are reported here, since the simulations with ABAQUS quadratic shell elements (i.e., STRI65 and S8R) failed to converge in the current deep drawing test. In the case of hexahedral elements (i.e., SHB20 and C3D20), the quarter of the circular blank is meshed with 255 quadratic elements, while 510 quadratic elements are used in the case of prismatic elements (i.e., SHB15 and C3D15). All simulations are performed using 
only a single element layer with three through-thickness integration points. All along the forming process, a constant holding force of $5.55 \mathrm{kN}$ is applied, and the friction coefficient between the sheet and the forming tools is taken equal to 0.1. Figure 37 illustrates the geometric shape of the formed ear on a quarter cup obtained with the SHB elements.

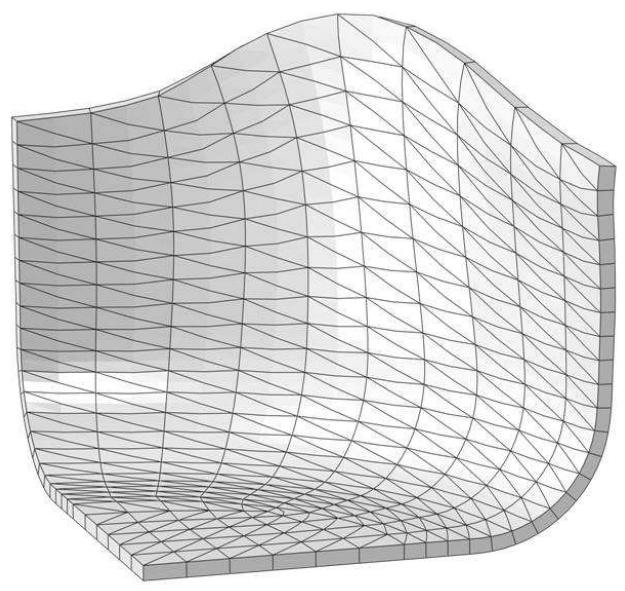

(a) $(255 \times 2) \times 1$ SHB15 elements

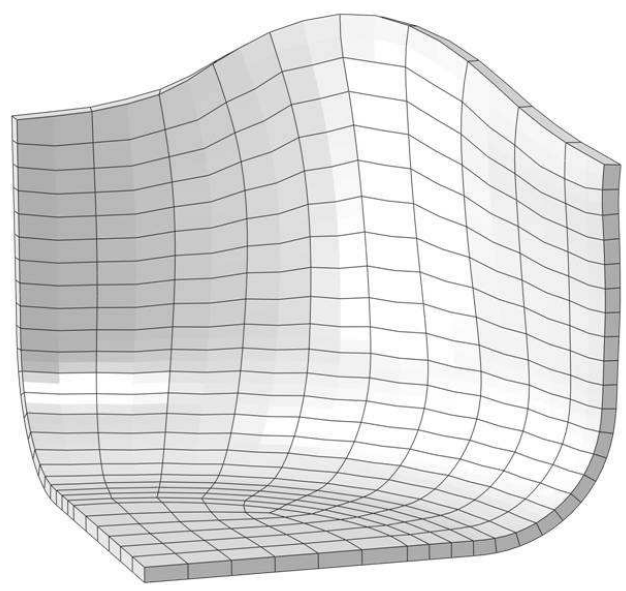

(b) $255 \times 1$ SHB20 elements

Fig. 37. Final deformed shape for a quarter cup.

The earing profile predictions given by the SHB elements and ABAQUS solid elements are reported in Fig. 38 along with the experimental measurements provided by Yoon et al. [52]. On the whole, it can be observed that both the shape and the height of the earing profiles predicted with the proposed SHB elements are in good agreement with the experimental results. More specifically, in the range around the experimental peak value, the results given by the SHB elements are the closest to the experimental heights, in comparison with ABAQUS predictions. However, the predicted cup heights are underestimated at $0^{\circ}$ and $90^{\circ}$ from the rolling direction, both with the SHB elements and with ABAQUS solid elements. Nevertheless, these predictions could be improved in future work by adopting more appropriate anisotropic non-quadratic yield functions for aluminum alloys (see, e.g., $[52,54,55]$ ), which are able to predict more than four earing profiles for the complete circular blank, as observed experimentally. 


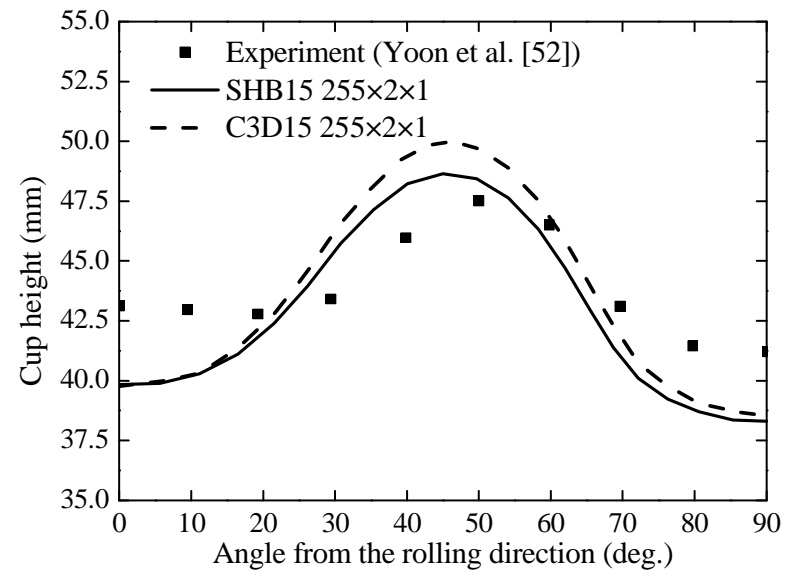

(a) prismatic elements

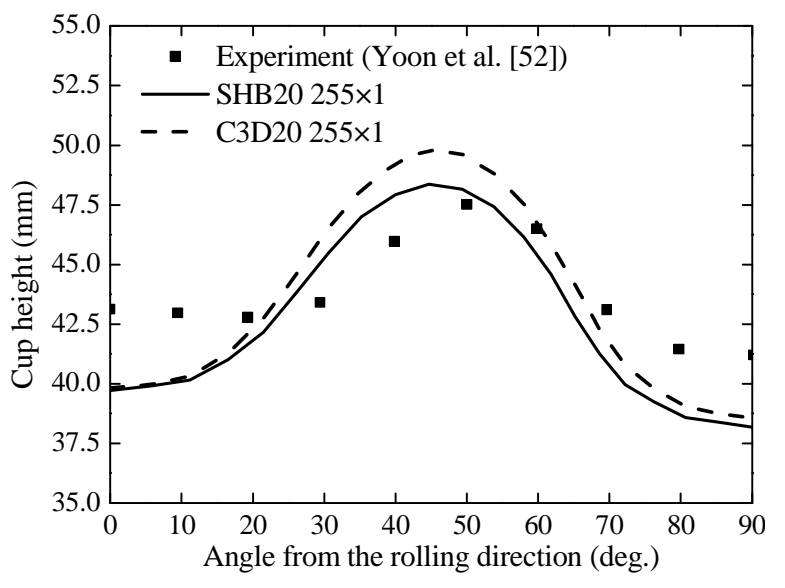

(b) hexahedral elements

Fig. 38. Predicted cup height profiles for the cylindrical cup.

\section{Conclusions}

In this work, two quadratic prismatic and hexahedral solid-shell elements denoted SHB15 and SHB20, respectively, have been proposed for the three-dimensional modeling of thin structures. With regard to earlier developments, the formulation of these elements has been extended in this paper to the framework of large strains and anisotropic plastic behavior. Based on a convenient fully three-dimensional framework, with only translational degrees of freedom and few throughthickness integration points, these elements appear to be very attractive, as they are able to accurately reproduce shell-like behavior using only a single element layer. These resulting SHB formulations have been implemented into the finite element code ABAQUS/Standard with the help of UEL subroutines. The present analysis mainly focuses on large-strain applications using fully three-dimensional anisotropic elasto-plastic constitutive equations.

The performance of the proposed SHB elements has been first evaluated on a series of selective linear and nonlinear benchmark tests. For each benchmark problem, the results given by the SHB elements have been compared with those yielded by their ABAQUS counterparts, including quadratic solid and shell elements, as well as with reference solutions taken from the literature. For all benchmark tests, the numerical results obtained with the SHB elements showed excellent agreement with the available reference solutions. Compared to state-of-the-art ABAQUS shell elements, the performance of the SHB elements is often comparable; however, in 
most numerical tests, ABAQUS quadratic solid elements (i.e., C3D15 and C3D20) require finer meshes (in the plane and through the thickness) to provide accurate solutions.

Then, the SHB elements have been applied to the simulation of four challenging sheet metal forming processes, in order to assess their capabilities in modeling complex problems involving large displacements and rotations, anisotropic large-strain plasticity and double-sided contact. Overall, three popular deep drawing processes and an incremental forming test have been considered. Various comparisons between the simulation results given by the SHB elements and the experimental measurements revealed that the proposed solid-shell elements are able to successfully model such complex forming processes, using only a single element layer with few through-thickness integration points. Furthermore, the SHB elements provided the closest results to the experimental data, when compared to ABAQUS quadratic solid elements. It is worth noting that the simulations of the sheet metal forming processes investigated using ABAQUS quadratic shell elements failed to converge, which reveals that these shell elements are not appropriate for handling double-sided contact in such complex forming processes.

Overall, the proposed quadratic SHB elements showed good capabilities in modeling various types of structural problems, with coarse meshes and few integration points through the thickness, whereas conventional quadratic solid elements required finer meshes to achieve accurate solutions. Also, the SHB elements represent an interesting alternative to traditional shell elements, especially for the simulation of complex problems involving double-sided contact, which are very common in sheet metal forming processes.

\section{References}

1. Zienkiewicz OC, Taylor RL, Too JM (1971) Reduced integration technique in general analysis of plates and shells. Int J Numer Methods Eng 3:275-290

2. Cho C, Park HC, Lee SW (1998) Stability analysis using a geometrically nonlinear assumed strain solid shell element model. Finite Elem Anal Des 29:121-135

3. Hauptmann R, Schweizerhof K (1998) A systematic development of 'solid-shell' element formulations for linear and non-linear analyses employing only displacement degrees of freedom. Int J Numer Methods Eng 42:49-69 
4. Puso MA (2000) A highly efficient enhanced assumed strain physically stabilized hexahedral element. Int J Numer Methods Eng 49:1029-1064

5. Sze KY, Yao LQ (2000) A hybrid stress ANS solid-shell element and its generalization for smart structure modelling Part I-solid-shell element formulation. Int J Numer Methods Eng 48:545-564

6. Abed-Meraim F, Combescure A (2002) SHB8PS-a new adaptive, assumed-strain continuum mechanics shell element for impact analysis. Comput Struct 80:791-803

7. Kim JH, Kim YH (2002) A three-node $\mathrm{C}^{0}$ ANS element for geometrically non-linear structural analysis. Comput Methods Appl Mech Eng 191:4035-4059

8. Alves de Sousa RJ, Cardoso RPR, Fontes Valente RA, Yoon JW, Grácio JJ, Natal Jorge RM (2005) A new one-point quadrature enhanced assumed strain (EAS) solid-shell element with multiple integration points along thickness: Part I-geometrically linear applications. Int J Numer Methods Eng 62:952-977

9. Parente MPL, Fontes Valente RA, Natal Jorge RM, Cardoso RPR, Alves de Sousa RJ (2006) Sheet metal forming simulation using EAS solid-shell finite elements. Finite Elem Anal Des 42:1137-1149

10. Reese S (2007) A large deformation solid-shell concept based on reduced integration with hourglass stabilization. Int J Numer Methods Eng 69:1671-1716

11. Cardoso RPR, Yoon JW, Mahardika M, Choudhry S, Alves de Sousa RJ, Fontes Valente RA (2008) Enhanced assumed strain (EAS) and assumed natural strain (ANS) methods for onepoint quadrature solid-shell elements. Int J Numer Methods Eng 75:156-187

12. Abed-Meraim F, Combescure A (2009) An improved assumed strain solid-shell element formulation with physical stabilization for geometric non-linear applications and elasticplastic stability analysis. Int J Numer Methods Eng 80:1640-1686

13. Schwarze M, Reese S (2009) A reduced integration solid-shell finite element based on the EAS and the ANS concept-Geometrically linear problems. Int J Numer Methods Eng 80:1322-1355 
14. Li LM, Peng YH, Li DY (2011) A stabilized underintegrated enhanced assumed strain solidshell element for geometrically nonlinear plate/shell analysis. Finite Elem Anal Des 47:511518

15. Edem IB, Gosling PD (2012) One-point quadrature ANS solid-shell element based on a displacement variational formulation Part I - Geometrically linear assessment. Comput Methods Appl Mech Eng 237-240:177-191

16. Flores FG (2013) Development of a non-linear triangular prism solid-shell element using ANS and EAS techniques. Comput Methods Appl Mech Eng 266:81-97

17. Pagani M, Reese S, Perego U (2014) Computationally efficient explicit nonlinear analyses using reduced integration-based solid-shell finite elements. Comput Methods Appl Mech Eng 268:141-159

18. Schwarze M, Vladimirov IN, Reese S (2011) Sheet metal forming and springback simulation by means of a new reduced integration solid-shell finite element technology. Comput Methods Appl Mech Eng 200:454-476

19. Salahouelhadj A, Abed-Meraim F, Chalal H, Balan T (2012) Application of the continuum shell finite element SHB8PS to sheet forming simulation using an extended large strain anisotropic elastic-plastic formulation. Arch Appl Mech 82:1269-1290

20. Trinh VD, Abed-Meraim F, Combescure A (2011) A new assumed strain solid-shell formulation "SHB6" for the six-node prismatic finite element. J Mech Sci Tech 25:23452364

21. Abed-Meraim F, Trinh VD, Combescure A (2013) New quadratic solid-shell elements and their evaluation on linear benchmark problems. Computing 95:373-394

22. Zienkiewicz OC, Taylor RL, Zhu JZ (2006) The Finite Element Method. Sixth ed., Elsevier Ltd

23. Hallquist JO (1983) Theoretical manual for DYNA3D. Report UC1D-19041, Lawrence Livermore National Laboratory, Livermore, CA, USA

24. Simo JC, Hughes TJR (1986) On the variation foundations of assumed strain methods. J Appl Mech 53:51-54 
25. Hill R (1948) A theory of the yielding and plastic flow of anisotropic metals. Proc. Roy. Soc. London A 193:281-297

26. Timoshenko S, Woinowsky-Krieger S (1959) Theory of plates and shells. Second ed., McGraw-Hill Book Co.

27. Peng X, Crisfield M (1992) A consistent co-rotational formulation for shells using the constant stress/constant moment triangle. Int J Numer Methods Eng 35:1829-1847

28. Sansour C, Bufler H (1992) An exact finite rotation shell theory, its mixed variational formulation and its finite element implementation. Int J Numer Methods Eng 34:73-115

29. Sze KY, Liu XH, Lo SH (2004) Popular benchmark problems for geometric nonlinear analysis of shells. Finite Elem Anal Des 40:1551-1569

30. Park HC, Cho C, Lee SW (1995) An efficient assumed strain element model with 6 dof per node for geometrically nonlinear shells. Int J Numer Methods Eng 38:4101-4122

31. Sze KY, Chan WK, Pian THH (2002) An eight-node hybrid-stress solid-shell element for geometric non-linear analysis of elastic shells. Int J Numer Methods Eng 55:853-878

32. Hsiao KM (1987) Nonlinear analysis of general shell structures by flat triangular elements. Comput Struct 25:665-675

33. Barut A, Madenci E, Tesslerb A (1997) Nonlinear analysis of laminates through a Mindlintype shear deformable shallow shell element. Comput Methods Appl Mech Eng 143:155173

34. Smoleński WM (1999) Statically and kinematically exact nonlinear theory of rods and its numerical verification. Comput Methods Appl Mech Eng 178:89-113

35. Mostafa M, Sivaselvan MV, Felippa CA (2013) A solid-shell corotational element based on ANDES, ANS and EAS for geometrically nonlinear structural analysis. Int J Numer Methods Eng 95:145-180

36. Betsch P, Stein E (1999) Numerical implementation of multiplicative elasto-plasticity into assumed strain elements with application to shells at large strains. Comput Methods Appl Mech Eng 179:215-245 
37. Fontes Valente RA, Alves de Sousa RJ, Natal Jorge RM (2004) An enhanced strain 3D element for large deformation elastoplastic thin-shell applications. Comput Mech 34:38-52

38. Cardoso RPR, Yoon JW (2005) One point quadrature shell element with through-thickness stretch. Comput Methods Appl Mech Eng 194:1161-1199

39. Wriggers P, Eberlein R, Reese S (1996) A comparison of three-dimensional continuum and shell elements for finite plasticity. Int J Solids Struct 33:3309-3326

40. Eberlein R, Wriggers P (1999) Finite element concepts for finite elastoplastic strains and isotropic stress response in shells: theoretical and computational analysis. Comput Methods Appl Mech Eng 171:243-279

41. Hauptmann R, Schweizerhof K, Doll S (2000) Extension of the 'solid-shell' concept for application to large elastic and large elastoplastic deformations. Int J Numer Methods Eng 49:1121-1141

42. Makinouchi A, Nakamachi E, Oñate E, Wagoner RH (1993) NUMISHEET'93, Proceedings of the 2nd International Conference and Workshop on Numerical Simulation of 3D Sheet Metal Forming Processes - Verification of Simulation with Experiment, Isehara, Japan

43. Park DW, Oh SI (2004) A four-node shell element with enhanced bending performance for springback analysis. Comput Methods Appl Mech Eng 193:2105-2138

44. Zhang DJ, Cui ZS, Ruan XY, Li YQ (2007) An analytical model for predicting springback and side wall curl of sheet after U-bending. Comput Mater Sci 38:707-715

45. Dvorkin EN, Bathe KJ (1984) A continuum mechanics based four-node shell element for general nonlinear analysis. Eng Computations 1:77-88

46. Bouffioux C, Eyckens P, Henrard C, Aerens R, Van Bael A, Sol H, Duflou JR, Habraken AM (2008) Identification of material parameters to predict Single Point Incremental Forming forces. Int J Plasticity 1:1147-1150

47. Sena JIV, Alves de Sousa RJ, Valente RAF (2010) Single point incremental forming simulation with an enhanced assumed strain solid-shell finite element formulation. Int $\mathbf{J}$ Mater Form 3:963-966 
48. Wriggers P (2006) Computational contact mechanics. Second ed., Springer.

49. Xu HJ, Liu YQ, Zhong W (2012) Three-dimensional finite element simulation of medium thick plate metal forming and springback. Finite Elem Anal Des 51:49-58

50. Yoon JW, Barlat F, Chung K, Pourboghrat F, Yang DY (2000) Earing predictions based on asymmetric nonquadratic yield function. Int J Plasticity 16:1075-1104

51. Yoon JW, Barlat F, Dick RE, Chung K, Kang TJ (2004) Plane stress yield function for aluminum alloy sheets part II: FE formulation and its implementation. Int J Plasticity $20: 495-522$

52. Yoon JW, Barlat F, Dick RE, Karabin ME (2006) Prediction of six or eight ears in a drawn cup based on a new anisotropic yield function. Int J Plasticity 22:174-193

53. Schwarze M, Vladimirov IN, Reese S (2010) A new continuum shell finite element for sheet metal forming applications. Int J Mater Form 3:919-922

54. Barlat F, Lege DJ, Brem JC (1991) A six-component yield function for anisotropic materials. Int J Plasticity 7:693-712

55. Barlat F, Brem JC, Yoon JW, Chung K, Dick RE, Lege DJ, Pourboghrat F, Choi SH, Chu E (2003) Plane stress yield function for aluminum alloy sheets - part 1: theory. Int J Plasticity 19:1297-1319 